\title{
Selective vulnerability of inhibitory networks in multiple sclerosis
}

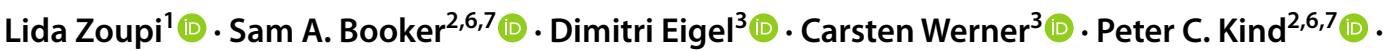

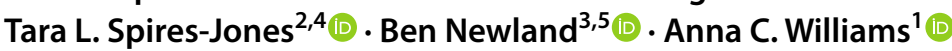

Received: 12 October 2020 / Revised: 21 December 2020 / Accepted: 22 December 2020 / Published online: 15 January 2021 (c) The Author(s) 2021

\begin{abstract}
In multiple sclerosis (MS), a chronic demyelinating disease of the central nervous system, neurodegeneration is detected early in the disease course and is associated with the long-term disability of patients. Neurodegeneration is linked to both inflammation and demyelination, but its exact cause remains unknown. This gap in knowledge contributes to the current lack of treatments for the neurodegenerative phase of MS. Here we ask if neurodegeneration in MS affects specific neuronal components and if it is the result of demyelination. Neuropathological examination of secondary progressive MS motor cortices revealed a selective vulnerability of inhibitory interneurons in MS. The generation of a rodent model of focal subpial cortical demyelination reproduces this selective neurodegeneration providing a new preclinical model for the study of neuroprotective treatments.
\end{abstract}

Keywords Multiple sclerosis $\cdot$ Neurodegeneration $\cdot$ Interneurons $\cdot$ Myelin $\cdot$ Synapses

Supplementary Information The online version contains supplementary material available at https://doi.org/10.1007/s0040 1-020-02258-z.

Anna C. Williams

anna.williams@ed.ac.uk

1 Centre for Regenerative Medicine, Institute for Regeneration and Repair, University of Edinburgh, Edinburgh EH16 4UU, UK

2 Centre for Discovery Brain Sciences, University of Edinburgh, Edinburgh EH8 9XD, UK

3 Leibniz-Institut Für Polymerforschung Dresden E.V, Max Bergmann Center of Biomaterials Dresden, Hohe Straße 6, 01069 Dresden, Germany

4 UK Dementia Research Institute, University of Edinburgh, Edinburgh EH8 9JZ, UK

5 School of Pharmacy and Pharmaceutical Sciences, Cardiff University, Cardiff CF10 3NB, UK

6 Simons Initiative for the Developing Brain, University of Edinburgh, Edinburgh EH8 9XD, UK

7 Patrick Wild Centre for Autism Research, University of Edinburgh, Edinburgh EH8 9XD, UK

\section{Introduction}

Multiple sclerosis (MS) is a chronic neuroinflammatory disease of the human central nervous system (CNS) characterised by inflammation, focal areas of demyelination and neurodegeneration. At the early stages of the disease, inflammatory cells target the myelin sheath that insulates and supports the axons. Current treatments that modulate this immune attack suppress the inflammatory demyelinating white matter lesions (as assessed by magnetic resonance imaging-MRI) and accompanying relapses, but ultimately fail to prevent neurodegeneration [16].

Neurodegeneration in MS is reported to consist of neurite transection, diffuse synaptic dysfunction and overall neuronal loss [1, 12, 36, 39, 46, 48]. Long term disability better correlates with brain atrophy on MRI rather than with white matter demyelinating lesion load, but the mechanisms leading to these changes remain largely unknown [7, 8, 22]. This limited understanding may contribute to the lack of successful therapies counteracting neurodegeneration. Thus, two fundamental questions need to be addressed: first, which neural components are primarily affected? Second, does neurodegeneration result directly from demyelination?

Given that cortical thinning/atrophy in MS is widespread, one may predict that all neuronal classes are potentially affected. However, in a recent transcranial magnetic 
stimulation study, motor dysfunction strongly correlates with increased excitatory and reduced inhibitory transmission in the motor cortex of MS patients, suggesting possible differential effects [47]. Although demyelination in MS is classically identified in the white matter, due to the relative ease of identification on MRI scans, it is well-recognised that demyelination is also common in the grey matter [3, $4,23,26]$. Focal demyelinated lesions occur in the grey matter, including in the cortex, disrupting neuronal signaling directly or via an inflammatory toxic environment [18, 31]. Differential effects of these lesions on various cortical neurons is possible due to spatial patterning, with lesions identified in both superficial (subpial lesions) and deeper cortical layers (intracortical, leukocortical lesions) [3, 4, 26]. Furthermore, the pattern of myelination of neuronal subtypes is variable, potentially increasing their susceptibility to neurodegeneration via demyelination: only some subtypes of cortical inhibitory interneurons are myelinated and while some pyramidal excitatory neuronal axons are fully myelinated along their length, others have discontinuous patterns of myelination, with large unmyelinated gaps between myelin sheaths [37, 38, 43-45, 51].

Notably, it is still controversial as to whether or to what extent neurodegeneration is caused by demyelination directly. Proponents indict the evidence that demyelination deprives the axon of insulation and essential metabolic supplies from the oligodendrocyte which can lead to axonal degeneration in animal models, via mitochondrial dysfunction and persistent oxidative stress [13, 49]. Supporting this, in chronic inactive demyelinated MS lesions, which by definition have little inflammation, there is slow axonal disintegration [27, 39]. However, opponents of this theory point out that demyelination in MS is also accompanied by (or driven by) inflammation, including cytotoxic $\mathrm{T}$ cells and macrophages, which can trigger acute axonal damage by releasing neurotoxic factors such as cytokines and reactive oxygen species outside the areas of demyelination and perhaps unconnected to it [18, 30-33]. Supporting this, is the widespread neurodegeneration seen in MS, which does not correlate with the number of demyelinated MS lesions at least in the white matter [7]. Furthermore, in the rodent model of experimental autoimmune encephalomyelitis (EAE), inflammation appears to mediate demyelination and neurodegeneration independently [48].

A better understanding of what neurodegeneration consists of in MS and the mechanism of damage, may aid the development of therapeutic strategies to limit it. To address this, we first asked if there is specific vulnerability of different neurons to neurodegeneration in MS brains. By neuropathological examination of the motor cortex of secondary progressive MS patients, compared to non-neurological controls, we found that inhibitory neurons are more susceptible to neurodegeneration. Next, to examine if grey matter demyelination leads to neurodegeneration, we generated a rodent model of focal cortical subpial demyelination that also shows selective inhibitory neuron and synapse loss and provided temporal evidence of demyelination-induced neurodegeneration. Our data support the idea that neurodegeneration can be secondary to demyelination irrespective of the presence of diffuse inflammation in the cortex, whilst developing a preclinical model for screening drugs to improve neuroprotection in MS patients.

\section{Materials and methods}

\section{Human tissue}

Post-mortem brain tissue (motor cortices) from MS patients and non-neurological controls were provided by a UK prospective donor scheme with full ethical approval from the UK Multiple Sclerosis Society Tissue Bank (MREC/02/2/39) and from the MRC-Edinburgh Brain Bank (16/ES/0084). MS diagnosis was confirmed by neuropathological means by F. Roncaroli (Imperial College London) and Prof. Colin Smith (Centre for Clinical Brain Sciences, Centre for Comparative Pathology, Edinburgh) and clinical history was provided by R. Nicholas (Imperial College London) and Prof. Colin Smith. Tables 1 and 2 include details on samples used for histological analysis and array tomography, respectively (Online Resource Table 1,2). Table 3 gives a detailed list of the tissue samples used for each individual analysis (Online Resource Table 3). For histological analysis, tissue blocks of $2 \mathrm{~cm} \times 2 \mathrm{~cm} \times 1 \mathrm{~cm}$ were collected, fixed, dehydrated and embedded in paraffin blocks. $4 \mu \mathrm{m}$ sequential sections were cut and stored at room temperature. Grey matter MS lesions were identified using anti-Proteolipid Protein (PLP) immunostaining (Online Resource Fig. 1a, b). The samples chosen had obviously myelinated and demyelinated areas on the same section. MS demyelinated areas refer to areas with distinct lesions in the upper cortical layers and MS myelinated to normal appearing myelinated cortical layers. For array tomography, $1 \mathrm{~cm}^{3}$ motor cortex blocks were collected upon autopsy and further dissected in $1 \mathrm{~mm} \times 1 \mathrm{~mm} \times 5 \mathrm{~mm}$ array tomography samples before processing. The mean age for all samples is 63.75 years (control) and 62.70 years (MS), subdivided into paraffin samples: 77.60 years (control) and: 58.78 years (MS) and AT samples: 49.90 years (control) and 67.40 years (MS). Overall, 23 male and 30 female samples were used (controls: 15 male and 5 female samples, MS: 8 male and 25 female samples). Each sample was assessed by a neuropathologist and no signs of confounding neurodegenerative diseases were seen in any sample. 


\section{Animals}

Mice were housed and used according to the standard UK Home Office regulations, under project license PADF15B79. PSD95-eGFP animals were a gift from S.G.N.Grant (Centre for Clinical Brain Sciences, University of Edinburgh) [50]. 8-10-week-old male mice were used for all experiments.

\section{Array tomography (AT)}

Dissected tissue blocks were processed as previously described [25]. Briefly, small tissue blocks containing all 6 cortical layers were fixed for $2-3 \mathrm{~h}$ in $4 \%$ PFA, $2.5 \%$ sucrose in $0.01 \mathrm{M}$ PBS and washed in $3.5 \%$ sucrose, $50 \mathrm{mM}$ glycine in $0.01 \mathrm{mM}$ PBS at $4{ }^{\circ} \mathrm{C}$ overnight. AT samples were then dehydrated in graded series of ethanol for $5 \mathrm{~min}$ each, followed by 5-min incubation with 50:50 ethanol $100 \%$ LRWhite (London Resin Medium grade, Agar scientific) and 100\% LRWhite alone. Samples were then left overnight at $4{ }^{\circ} \mathrm{C}$ in LRWhite for complete infiltration, transferred to gelatin capsules filled with cold LRWhite and polymerized at $52{ }^{\circ} \mathrm{C}$ overnight. Tissue was sectioned into ribbons of $70 \mathrm{~nm}$ serial sections (30-40 sections/ribbon) with an ultramicrotome (Leica Ultracut) using a Ultra Jumbo Diamond Knife $35^{\circ}$ (Diatome) and mounted in gelatin covered coverslips that were allowed to air dry before immunohistochemistry.

\section{LPC-induced cortical demyelinating lesion in cortex}

In 8-10-week-old C57BL/6 or PSD95-eGFP anesthetized male mice; a craniotomy was made above the left motor cortex. Briefly, using a $0.6 \mathrm{~mm}$ dental drill tip and low drilling speed we thinned the skull above the M1 and M2 areas ( $2 \mathrm{~mm} \varnothing)$ avoiding brain overheating. When the skull was adequately thinned saline application allows for the removal of the cranial top avoiding damage to the underlying brain tissue. Macroporous, poly(ethylene glycol) (PEG) based cylindrical scaffolds (cryogel) were synthesized as previously reported [14] to dimensions $2 \mathrm{~mm} \varnothing \times 0.5 \mathrm{~mm}$ depth and loaded with either PBS (control) or $10 \mathrm{mg} / \mathrm{mL} \mathrm{L-} \alpha-$ Lysophosphatidylcholine (LPC, L4129, Sigma-Aldrich) and placed onto the exposed cortical surface. The skin was subsequently sutured back over the cryogel and the animals were left to recover. The release properties of LPC from the $2 \mathrm{~mm} \emptyset \times 0.5 \mathrm{~mm}$ cryogel showed successful release of $80 \%$ of the total LPC amount within $48 \mathrm{hrs}$ and $100 \%$ by $120 \mathrm{hrs}$ (Online Resource Fig. 5). Two time points were chosen for the present study ( 2 and 3 weeks post-surgery), characterized by the presence of focal demyelination in the superficial layers of the motor cortex. Mice were subsequently perfused with 4\% PFA (Sigma-Aldrich), the brain tissue was harvested, cryoprotected in $30 \%$ sucrose, frozen in 2-methylbutane (Sigma-Aldrich) and stored at $-80{ }^{\circ} \mathrm{C}$.

\section{Immunohistochemistry}

\section{Human post-mortem brain tissue}

Paraffin sections were rehydrated and microwaved for $15 \mathrm{~min}$ in Vector Unmasking Solution for antigen retrieval (H-3300, Vector). For chromogenic immunohistochemistry, endogenous peroxidase and alkaline phosphatase activities were blocked for 10 min with Bioxal solution (SP-6000, Vector). Sections were then blocked with 2.5\% normal horse serum (S-2012, Vector) for $1 \mathrm{~h}$ at room temperature. Primary antibodies were incubated in antibody diluent solution $(003,118$, Thermo Fisher Scientific), overnight at $4{ }^{\circ} \mathrm{C}$ in a humidified chamber. The next day, horse peroxidase or alkaline phosphatase-conjugated secondary antibodies (Vector) were applied for $1 \mathrm{~h}$ at room temperature. Staining was developed with either DAB peroxidase substrate kit or alkaline phosphatase substrate kit (both from Vector) according to the manufacturer's instructions.

For immunofluorescence, sections were incubated with Autofluorescent Eliminator Reagent (2160, MERCKMillipore) for $1 \mathrm{~min}$ and briefly washed in $70 \%$ ethanol after antigen retrieval. The sections were subsequently incubated with Image-iT® FX Signal Enhancer (I36933, Thermo Fisher Scientific) for $30 \mathrm{~min}$ at room temperature, washed and blocked for $1 \mathrm{~h}$ with $10 \%$ normal horse serum, $0.3 \%$ Triton-X in PBS. Primary antibodies were diluted in antibody diluent solution (as above) and incubated overnight at $4{ }^{\circ} \mathrm{C}$ in a humidified chamber. The next day sections were incubated with Alexa Fluor secondary antibodies (Thermo Fischer Scientific, 1:1000) for $1 \mathrm{~h}$ at room temperature and counterstained with Hoechst for nuclear visualization. All slides were mounted using Mowiol mounting medium (475,904, MERCK- Millipore).

\section{Array tomography}

Dried ribbons were incubated in $50 \mathrm{mM}$ glycine in $0.01 \mathrm{M}$ PBS for $5 \mathrm{~min}$, washed in $3.5 \%$ sucrose, $50 \mathrm{mM}$ glycine in $0.01 \mathrm{mM}$ PBS and blocked with $0.1 \%$ BSA, $0.05 \%$ Tween20 in Tris Buffered Saline solution (TBS) for $30 \mathrm{~min}$ at room temperature. Primary antibodies were diluted in blocking solution (all antibodies 1:50 dilution) and placed on ribbons overnight at $4{ }^{\circ} \mathrm{C}$. The following day ribbons were washed in TBS and incubated with secondary antibodies diluted in blocking solution with $0.01 \mathrm{mg} /$ $\mathrm{mL}$ DAPI for $30 \mathrm{~min}$ at room temperature (all secondary antibodies diluted 1:100). Finally, ribbons were washed 
and mounted on microscope slides with Shandon Immunomount (Thermo Scientific).

\section{Mouse tissue}

$10 \mu \mathrm{m}$ thick cryosections were briefly washed in PBS and microwaved for $15 \mathrm{~min}$ in Vector Unmasking Solution for antigen retrieval (H-3300, Vector) before blocking with $10 \%$ normal horse serum, $0.3 \%$ Triton-X in $1 \mathrm{xPBS}$ for $1 \mathrm{~h}$ at room temperature. Primary antibodies were diluted in antibody diluent $(003,118$, Thermo Fisher Scientific) and sections were incubated overnight at $4{ }^{\circ} \mathrm{C}$ in a humidified chamber. The following day, cryosections were incubated with Alexa Fluor secondary antibodies (Thermo Fischer Scientific, 1:1000) for $1 \frac{1 / 2}{2} \mathrm{hrs}$ at room temperature and counterstained with Hoechst for nuclear visualization. All slides were mounted using Mowiol mounting medium (475,904, MERCK- Millipore).

\section{Image acquisition and analysis.}

\section{Human post-mortem tissue}

Entire sections were imaged using the Zeiss AxioScan Slide scanner or the Vectra ${ }^{\circledR}$ Polaris ${ }^{\mathrm{TM}}$ Automated Quantitative Pathology Imaging System. All quantifications were performed using Zeiss Zen lite imaging software and QuPath open source software [2].

For cell density quantification 5-10 fields were chosen that included all cortical layers or L2/3 only, manually quantified and presented as cells $/ \mathrm{mm}^{2}$ or cells $/ \mathrm{cm}^{2}$. GM thickness was manually quantified by measuring the distance from the cortical surface to the lower edge of cortical layer six. At least 20 different measurements were obtained per section. Axonal measurements were obtained after double blinded quantification of SMI312-possitive axons as previously described [24]. Briefly, the relative axonal density crossing $100 \mu \mathrm{m}$ line was measured from 10-20 different areas depending on the section size.

For synaptic density quantification, 5-6 stacks $(184.58 \mu \mathrm{m} \times 184.58 \mu \mathrm{m}$ each) from layer $2 / 3 \quad$ (L2/3) of the motor cortex were obtained using high resolution confocal microscopy (Leica TCS SP8; $3144 \times 3144$ resolution, $150 \mathrm{~nm}$ optical z-step, $2 \mu \mathrm{m}$ total thickness). Each field was subdivided in $10 \mu \mathrm{m} \times 10 \mu \mathrm{m}$ regions of interest covering the area of the neuropil avoiding cell bodies and blood vessels. Images were cropped and segmented using automated local thresholding Fiji (Fiji, RRID:SCR_002285) algorithms. Segmentation parameters were exclusive for each channel but the same for all sections. To avoid false positive signal, objects that were not present in at least two consecutive sections were removed. Quantification of adjacent pre and post synaptic objects (object centers within $1 \mu \mathrm{m}$ distance) in the tissue volume was obtained using an in-house MATLAB algorithm. Values were averaged for each animal and presented as synapses $/ \mathrm{mm}^{3}$.

\section{Array tomography}

Images were obtained using a Zeiss AxioImager Z2 epifluorescent microscope with a CoolSnap digital camera and AxioImager software with array tomography macros (Carl Zeiss, Ltd, Cambridge UK). A tile scan of the ribbon is initially taken in low magnification followed by the generation of a position list that outlines the area of the ribbon. Serial high-resolution images are then taken with a $63 \times 1.4 \mathrm{NA}$ Plan Apochromat objective and aligned using MultiStackReg 1.4 Fiji plugin (Fiji, RRID:SCR_002285) [25]. Two to five image stacks were captured per tissue block per case. Synaptic quantification was performed as described above.

\section{Mouse tissue}

All sections were imaged using a Leica TCS SP8 confocal microscope. For cell density quantification 3-9 fields (150 $\mu \mathrm{m} \times 150 \mu \mathrm{m}$ each) were obtained from each section depending on the lesion size. Total number of cells was quantified in the lesion and perilesion area and represented as cells $/ \mathrm{mm}^{2}$. Perilesion area was defined as the area spanning $150 \mu \mathrm{m}$ from the lesion border. 3-5 sections were analyzed per animal and the numbers were averaged for each animal. For signal area quantification, the same size and number of areas were thresholded and automatically quantified using in-house Fiji macros (Fiji, RRID:SCR_002285).

Synaptic density quantification was done similarly to human tissue. 3 stacks $(184.58 \mu \mathrm{m} \times 184.58 \mu \mathrm{m}$ each $)$ from L2/3 of the motor cortex were obtained using high resolution confocal microscopy $(3144 \times 3144$ resolution, $150 \mathrm{~nm}$ optical z-step, $2 \mu \mathrm{m}$ total thickness). 3-5 sections were analyzed per animal. Quantification of adjacent pre and post synaptic objects (object centers within $0.5 \mu \mathrm{m}$ distance) in the tissue volume was obtained using an in-house MATLAB algorithm. Values were averaged for each animal and presented as synapses $/ \mathrm{mm}^{3}$.

Images were randomised using the File randomizer Fiji (Fiji, RRID:SCR_002285) macro prior to analysis.

Illustrations created with BioRender.com.

\section{In vitro slice electrophysiology}

Slices containing LPC lesion and PBS control neurons were prepared from mice which had been implanted with cryogels overlaying the motor cortex (M1), as previously described [5, 6]. Briefly, mice were sedated with isofluorane, decapitated and their brains rapidly removed and placed in ice-cold sucrose-modified artificial cerebrospinal fluid 
(sucrose-ACSF; in mM: $87 \mathrm{NaCl}, 2.5 \mathrm{KCl}, 25 \mathrm{NaHCO}_{3}$, $1.25 \mathrm{NaH}_{2} \mathrm{PO}_{4}, 25$ glucose, 75 sucrose, $7 \mathrm{MgCl}_{2}, 0.5 \mathrm{CaCl}_{2}$ ); saturated with carbogen $\left(95 \% \mathrm{O}_{2} / 5 \% \mathrm{CO}_{2}\right) .300 \mu \mathrm{m}$ coronal brain slices were then sliced on a vibratome (VT1200S, Leica, Germany) covering the region that was overlain by the cryogel, as assessed by eye during dissections, then transferred to submerged holding chambers filled with sucrose-ACSF at $35^{\circ} \mathrm{C}$ for $30 \mathrm{~min}$, then room temperature until needed.

Whole-cell patch-clamp recordings were performed to record miniature EPSCs and IPSCs. Slices were transferred to a submerged recording chamber which was perfused with ACSF (in mM: $125 \mathrm{NaCl}, 2.5 \mathrm{KCl}, 25 \mathrm{NaHCO}_{3}$, $1.25 \mathrm{NaH}_{2} \mathrm{PO}_{4}, 25$ glucose, $1 \mathrm{MgCl}_{2}, 2 \mathrm{CaCl}_{2}$, containing $500 \mathrm{nM}$ tetrodotoxin, TTX) and bubbled with carbogen, at a rate of 4-6 $\mathrm{mL} \cdot \mathrm{min}^{-1}$, recordings were maintained at $30 \pm 1{ }^{\circ} \mathrm{C}$ with an inline Peltier heating device (Scientifica, Brighton, UK). Slices were first visualised under low-power magnification $(4 \times$ Objective 0.2 NA, Neoplan, Olympus, Japan) with infrared differential-inference contrast microscopy using a digital camera (SciCamPro, Scientifica, UK) mounted on an upright microscope (SliceScope, Scientifica, UK) to the dorsal pole of the slice. Neurons were identified for recording with a high-power $40 \times$ water-immersion objective lens (1.0 N.A., Olympus, Japan) and chosen based on having an ovoid soma located in the upper region of L2, with an apical dendrite oriented towards the pial surface. Recording pipettes were pulled from borosilicate glass capillaries $(1.7 \mathrm{~mm}$ outer/1 mm inner diameter, Harvard Apparatus, UK) on a horizontal electrode puller (P-97, Sutter Instruments, CA, USA) and filled with a Cs-gluconate based intracellular solution (in mM 140 Cs-gluconate, $3 \mathrm{CsCl}, 0.2$ EGTA, 10 HEPES, $2 \mathrm{Na}_{2} \mathrm{ATP}, 2 \mathrm{MgATP}, 0.3$ $\mathrm{Na}_{2} \mathrm{GTP}, 10 \mathrm{Na}_{2}$ Phosphocreatine, 2.7 Biocytin, 5 QX-314. $\mathrm{Cl}, \mathrm{pH}=7.4,290-310 \mathrm{mOsm}$ ), which gave 3-5 M $\mathrm{M}$ resistance electrodes and a measured $\mathrm{Cl}^{-}$reversal of $-74 \mathrm{mV}$ under these recording conditions. Liquid junction potential was not corrected. Cells were rejected from further recording, if they required more than 200pA current injection to maintain a $-70 \mathrm{mV}$ holding potential, series resistance $>30$ $\mathrm{M} \Omega$, or series resistance changed by more than $20 \%$. All recording was performed with a Multiclamp 700B amplifier (Molecular Devices, CA, USA) and filtered online at $2 \mathrm{kHz}$ with the amplifiers 4-pole Bessel Filter and digitised at $20 \mathrm{kHz}$ (Digidata1550B, Molecular Devices, CA, USA).)

All in the presence of $500 \mathrm{mM}$ TTX, mEPSCs were recorded from $-70 \mathrm{mV}$ voltage clamp. $5 \mathrm{~min}$ of continuous recording were performed with series resistance measured before and after this period. Following mEPSC recordings, the membrane potential was switched to between 0 and $5 \mathrm{mV}$ - depending on measured $\mathrm{mEPSC}$ reversal — and 5 min of mIPSCs recorded. Following recording, the series resistance was measured again to confirm recording stability.
Out-side out patches were then performed on recorded neurons, to seal the included biocytin within cells. A further 2-3 cells were recorded from each slice and then slices fixed in 4\% PFA in $0.1 \mathrm{M} \mathrm{PB}$ overnight, then transferred to PBS until processed for histology.

mPSCs were detected offline using a template fitting approach, whereby exemplary mini-PSCs were fit with a triexponential curve [10], with thresholds of 4-7 imposed for detection. Following signal extraction, individual traces were excluded for analysis if they failed to exceed the threshold of $3 *$ SD of the baseline noise. EPSCs were detected as inward currents and ISPCs as outward currents. Traces were collected in pCLAMP 9 (Molecular Devices, CA, USA) and stored on a desktop computer. All analysis of electrophysiological data was performed offline using the Stimfit electrophysiological package [21]. All data collection and analysis were performed blind to treatment group.

\section{Results}

\section{Selective loss of myelinated interneurons and inhibitory synapses in the MS motor cortex.}

To assess the effects of MS on cortical structure, we first examined gross neurodegeneration in the motor cortex of post-mortem secondary progressive MS tissue and non-neurological controls. Grey matter (GM) thickness, measured as the distance between layers 1 to 6 , was similar between control and MS samples (Online Resource Fig. 1c). However, there was significant axonal loss in the MS motor cortex, as quantified using the pan-axonal marker SMI312 (Online Resource Fig. 1d,e and f), in accordance with studies reporting broad axonal loss in MS GM.

By their location in L2/3, these lost axons are either cortico-cortical projection axons, excitatory axon collaterals or inhibitory interneuron axons. Therefore, we next checked for changes in density of excitatory and inhibitory synapses between control and MS motor cortices, assuming overlap of pre- and post-synaptic puncta as evidence of a functional synapse. Using confocal microscopy and array tomography, we stained sections for the pre-synaptic marker synapsin1 (SYN1) and the excitatory post-synaptic marker post-synaptic density protein 95 (PSD95) (Fig. 1a, b, Online Resource Fig. 2a, b and c) or the inhibitory pre-synaptic marker vesicular GABA transporter (VGAT) and post-synaptic marker GEPHYRIN (Fig. 1e, f). Synapses were quantified in L2/3 of the cortex where the majority of cortico-cortical connections are formed, using automated methods previously described [25]. Both methods showed that there is no difference in the density of excitatory synapses or in individual pre- and post-synaptic components in the MS motor cortex when compared to controls (Fig. 1c and d \& Online Resource Fig. 2d). However, we 


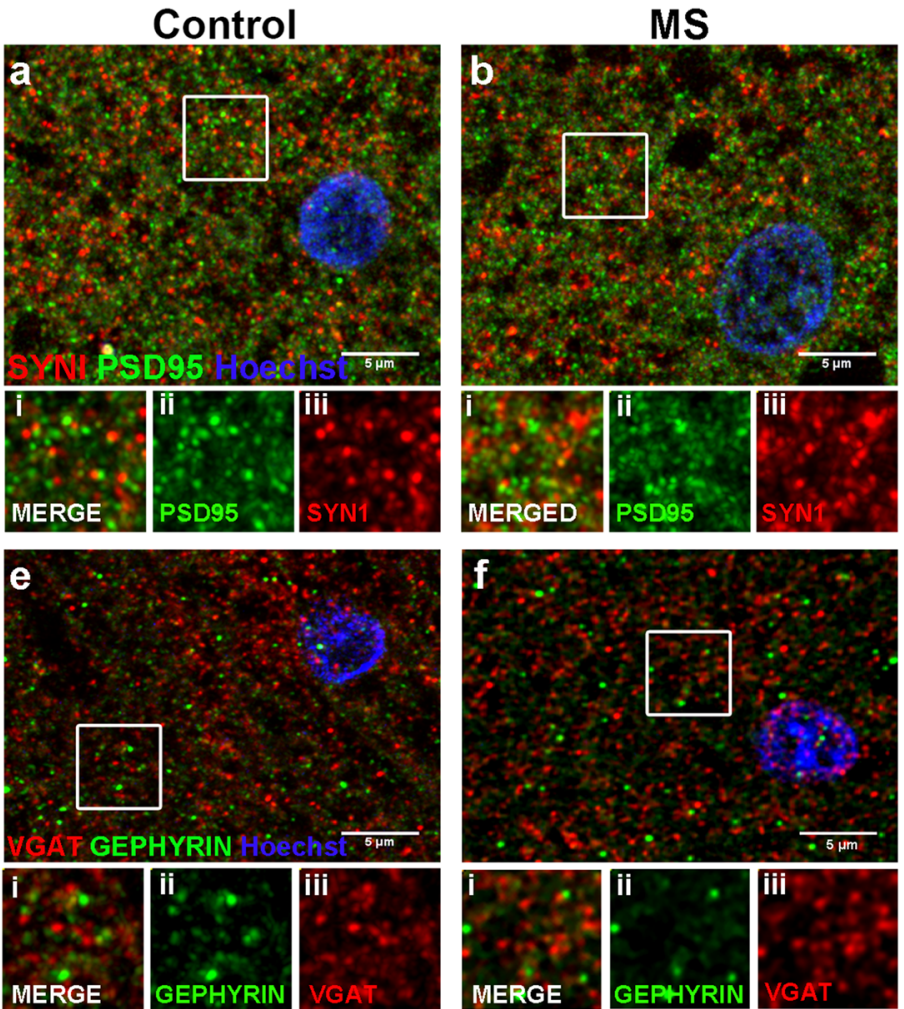

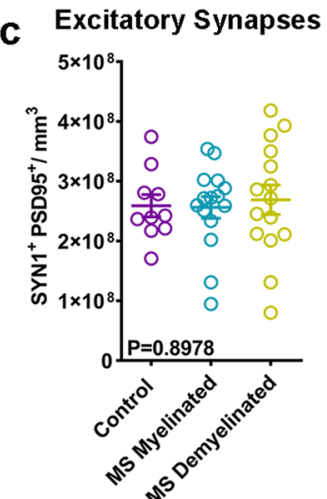

g Inhibitory Synapses

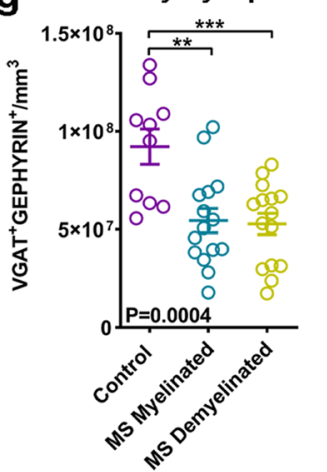

d Single Markers (Excitation)

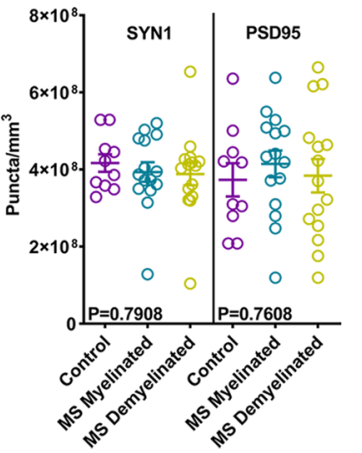

h Single Markers (Inhibition)

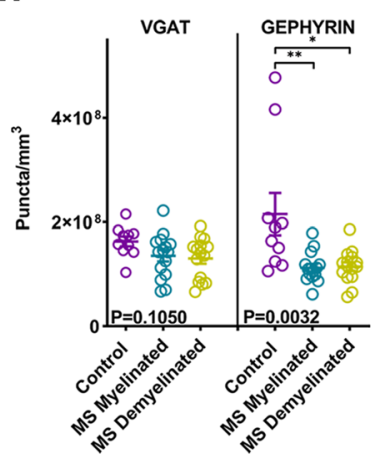

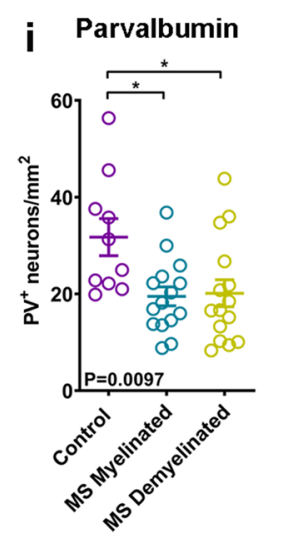

k Calbindin
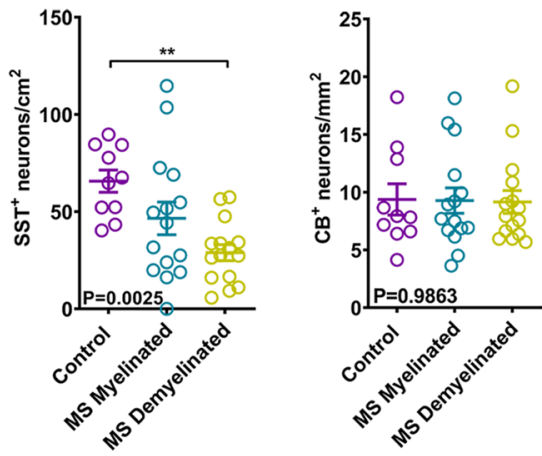
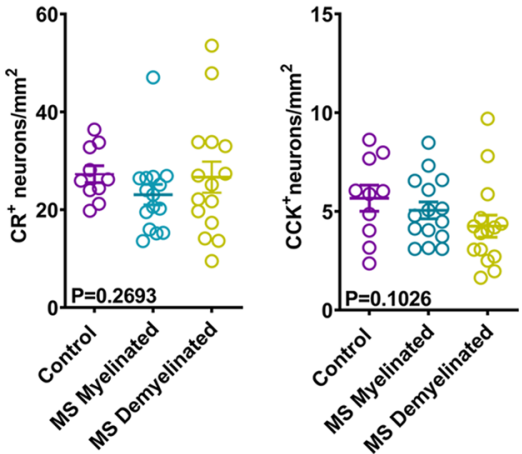

n Motor neurons observed a significant $~ 58 \%$ reduction in inhibitory synapses in MS motor cortices when compared to controls (Fig. 1g), attributed primarily to a significant reduction of post-synaptic components (Fig. 1h).

We then asked if these changes in inhibitory synapse density can be attributed to a selective loss of inhibitory interneurons. As not all interneurons are myelinated, we investigated whether there was a differential effect on interneuron subtypes. To do this, we immunolabelled cortical slices using antibodies against the calcium binding proteins parvalbumin (PV), calretinin (CR), calbindin (CB), or the neuropeptides somatostatin (SST) and cholecystokinin (CCK). We found a significant reduction in the density of PV expressing (PV +) and SST-expressing (SST +) interneurons (which usually have myelinated axons) (Fig. 1i, j
\& Online Resource Fig. 1g, h, I and j), but no change in the density of CRL, CCK and CB expressing subtypes, which typically have unmyelinated axons (Fig. 1k, 1 and $\mathrm{m} \&$ Online Resource Fig. $1 \mathrm{k}, 1, \mathrm{~m}, \mathrm{n}$, and p). In accordance with the lack of change of excitatory synaptic densities, there was no difference in the densities of excitatory motor neurons (using an antibody against the LIM-homeobox domain markers Islet1/2) (Fig. 1n \& Online Resource Fig. 1q, r) between control and MS cortex indicating that they survive even in late progressive MS. To assess changes in the overall neuronal densities in MS samples or a possible effect on the CUX2-positive population of projection neurons as previously reported by Schirmer et al.[40], we used the pan neuronal marker NEUN and the upper cortical layer marker CUX2 respectively (Online Resource Fig. 1s, t, w, x). 
४Fig. 1 a and b Immunohistochemistry of control (a) and MS (b) motor cortex (L2/3) for SYN1 (red), PSD95 (green), with Hoechst (blue). Scale bar: $5 \mu \mathrm{m}$. Inset images of boxed areas in a or b showing merged (i) and single channels of the synaptic proteins PSD95 (ii) and SYN1 (iii). c Quantification of SYN1+/PSD95 + excitatory synapses in L2/3 neuropil of control and MS myelinated or demyelinated motor cortex (Control: mean $2.589 \mathrm{e}+008 \pm 1.860 \mathrm{e}+007$ SEM synapses $/ \mathrm{mm}^{3}, N=10$, MS Myelinated: mean $2.562 \mathrm{e}+008 \pm 1.813 \mathrm{e}+007$ SEM synapses $/ \mathrm{mm}^{3}, \quad N=15, \quad$ MS Demyelinated: mean $2.690 \mathrm{e}+008 \pm 2.462 \mathrm{e}+007$ SEM synapses $/ \mathrm{mm}^{3}, N=15$; each point is a patient, One-way ANOVA). d Quantification of SYN1+presynaptic or PSD95 + post-synaptic puncta in L2/3 neuropil of control and MS myelinated or demyelinated motor cortex (SYN1 +: Control mean: $4.167 \mathrm{e}+008 \pm 2.275 \mathrm{e}+007 \mathrm{SEM}$ puncta $/ \mathrm{mm}^{3}, N=10$, MS Myelinated: mean $3.933 \mathrm{e}+008 \pm 2.533 \mathrm{e}+007$ SEM puncta $/ \mathrm{mm}^{3}$, $N=15$, MS Demyelinated: mean $3.881 \mathrm{e}+008 \pm 2.907 \mathrm{e}+007$ SEM puncta $/ \mathrm{mm}^{3}, N=15$; Kruskal-Wallis test; PSD95 +: Control mean: $3.731 \mathrm{e}+008 \pm 4.300 \mathrm{e}+007 \mathrm{SEM}$ puncta $/ \mathrm{mm}^{3}, N=10$, MS Myelinated: mean $4.144 \mathrm{e}+008 \pm 3.467 \mathrm{e}+007 \mathrm{SEM}$ puncta $/ \mathrm{mm}^{3}, N=15$, MS Demyelinated: mean $3.837 \mathrm{e}+008 \pm 4.337 \mathrm{e}+007$ SEM puncta/ $\mathrm{mm}^{3}, N=15$; each point is a patient; One-way ANOVA). e-f Immunohistochemistry of control (e) and MS (f) motor cortex (L2/3) for VGAT (red) GEPHYRIN (green) with Hoechst (blue). Scale bar: $5 \mu \mathrm{m}$. Inset images of boxed areas in e or f showing merged (i) and single channels of the synaptic proteins GEPHYRIN (ii) and VGAT (iii). g Quantification of VGAT +/GEPHYRIN + inhibitory synapses in L2/3 neuropil of control and MS myelinated or demyelinated motor cortex (Control: mean 9.217e $+007 \pm 8.997 \mathrm{e}+006$ SEM synapses/ $\mathrm{mm}^{3}, \quad N=10$, MS Myelinated: mean $5.444 \mathrm{e}+007 \pm 6.163 \mathrm{e}+006$ SEM synapses $/ \mathrm{mm}^{3}, \quad N=15, \quad$ MS Demyelinated: mean $5.274 \mathrm{e}+007 \pm 5.401 \mathrm{e}+006 \mathrm{SEM}$ synapses $/ \mathrm{mm}^{3}, N=15$; each point is a patient, One-way ANOVA). h Quantification of VGAT + presynaptic or GEPHYRIN + post-synaptic puncta in L2/3 neuropil of control and MS myelinated or demyelinated motor cortex (VGAT + : Control mean: $1.630 \mathrm{e}+008 \pm 9.452 \mathrm{e}+007 \mathrm{SEM}$ puncta $/ \mathrm{mm}^{3}, N=10$, MS Myelinated: mean $1.354 \mathrm{e}+008 \pm 1.087 \mathrm{e}+007$ SEM puncta/ $\mathrm{mm}^{3}, N=15$, MS Demyelinated: mean $1.302 \mathrm{e}+008 \pm 1.006 \mathrm{e}+007$ SEM puncta/ $\mathrm{mm}^{3}, N=15$; One-way ANOVA; GEPHYRIN +: Control mean: $2.151 \mathrm{e}+008 \pm 4.040 \mathrm{e}+007 \mathrm{SEM}$ puncta $/ \mathrm{mm}^{3}, \quad N=10$, MS Myelinated: mean $1.124 \mathrm{e}+008 \pm 7.416 \mathrm{e}+007$ SEM puncta/ $\mathrm{mm}^{3}, N=15$, MS Demyelinated: mean $1.148 \mathrm{e}+008 \pm 8.094 \mathrm{e}+007$ SEM puncta $/ \mathrm{mm}^{3}, N=15$; each point is a patient; Kruskal-Wallis test). i: Quantification of PV + interneuron density in all six cortical layers in control and MS myelinated or demyelinated motor cortex (Control: mean $31.74 \pm 3.834$ SEM neurons $/ \mathrm{mm}^{2}, N=10$, MS Myelinated: mean $19.49 \pm 1.961$ SEM neurons $/ \mathrm{mm}^{2}, N=15$, MS Demyelinated: mean $20.14 \pm 2.782$ SEM neurons $/ \mathrm{mm}^{2}, N=15$; each point is a patient, One-way ANOVA). $\mathbf{j}$ Quantification of SST + interneuron density in all six cortical layers in control and MS myelinated or demyelinated motor cortex (Control: mean 65.68 \pm 5.713 SEM neurons $/ \mathrm{cm}^{2}, N=10$, MS Myelinated: mean 46.55 \pm 8.406 SEM neurons/ $\mathrm{cm}^{2}, N=15$, MS Demyelinated: mean $28.95 \pm 4.123$ SEM neurons/ $\mathrm{cm}^{2}, N=15$; each point is a patient, One-Way ANOVA). k Quantification of calbindin $(\mathrm{CB})+$ interneuron density in all six cortical layers in control and MS myelinated or demyelinated motor cortex (Control: mean $9.377 \pm 1.353$ SEM neurons $/ \mathrm{mm}^{2}, N=10$, MS Myelinated: mean $9.276 \pm 1.101$ SEM neurons $/ \mathrm{mm}^{2}, N=15$, MS Demyelinated: mean $9.156 \pm 0.985 \mathrm{SEM}$ neurons $/ \mathrm{mm}^{2}, N=15$; each point is a patient, Kruskal-Wallis test). 1 Quantification of calretinin (CR) + interneuron density in all six cortical layers in control and MS myelinated or demyelinated motor cortex (Control: mean 27.27 \pm 1.731 SEM neurons $/ \mathrm{mm}^{2}, N=10$, MS Myelinated: mean 23.08 \pm 2.096 SEM neurons/ $\mathrm{mm}^{2}, N=15$, MS Demyelinated: mean 26.66 \pm 3.178 SEM neurons/ $\mathrm{mm}^{2}, N=15$; each point is a patient, Kruskal-Wallis test). $\mathbf{m}$ Quantification of cholecystokinin (CCK) + interneuron density in all six cortical layers in control and MS myelinated or demyelinated motor cortex
(Control: mean 5.668 \pm 0.6581 SEM neurons $/ \mathrm{mm}^{2}, N=10$, MS Myelinated: mean $5.052 \pm 0.4246$ SEM neurons $/ \mathrm{mm}^{2}, N=15$, MS Demyelinated: mean $4.251 \pm 0.5581$ SEM neurons $/ \mathrm{mm}^{2}, N=15$; each point is a patient, Kruskal-Wallis test). n Quantification of ISLET1/2+ motor neuron density in all six cortical layers in control and MS myelinated or demyelinated motor cortex (Control: $258.6 \pm 10.95$ SEM neurons/ $\mathrm{mm}^{2}, N=10$, MS Myelinated: mean $229.7 \pm 18.63$ SEM neurons $/ \mathrm{mm}^{2}$, $N=15$, MS Demyelinated: mean $229.4 \pm 18.84$ SEM neurons $/ \mathrm{mm}^{2}$, $N=15$; each point is a patient, One-way ANOVA)

We did not observe a significant reduction in either case (Online Resource Fig. 1u, v, y).

This selective reduction of two interneuron subtypes, $\mathrm{PV}+$ and SST +, which are normally myelinated, suggested an increased susceptibility of these to neurodegeneration in MS. These changes were present in areas of motor cortex that appeared to possess typical levels of myelination (normal appearing grey matter-NAGM) and those that were clearly demyelinated (Fig. 1i,j), with the caveat that NAGM areas show similar axonal loss to the demyelinated areas (Online Resource Fig. 1f) and so cannot be considered as normal healthy tissue. No correlation was found between the extent of loss of interneurons, inhibitory synapses or axons and patient characteristics such as age at the time of death, post-mortem interval, disease duration and years of progressive disease. These results clearly show that specific interneuron subtypes are susceptible to neurodegeneration in the cortex of MS patients. Hence, we next tested whether cortical demyelination drives this pattern of neurodegeneration by developing an animal model.

\section{Loss of inhibitory neurons and synapses in mouse cortical demyelinated lesions}

To investigate whether this selective neurodegeneration is driven by demyelination, we developed a novel, toxininduced mouse model of focal subpial cortical demyelination. Using a macroporous poly(ethylene glycol) (PEG) based cylindrical scaffold (termed cryogel), we delivered the demyelinating toxin lysophosphatidylcholine (LPC) directly onto the surface of the motor cortex (Fig. 2a). We have previously shown that this method generates focal cortical lesions ex vivo, in organotypic slice cultures [14]. In vivo, a single subpial focal demyelinating lesion was formed below the LPC-loaded cryogel at two weeks postsurgery (Fig. 2e, h) as shown by the lack of myelin basic protein (MBP) signal at the lesion site and reduced signal in the perilesion area in the upper cortical layers (defined as the area extending $150 \mu \mathrm{m}$ from the lesion border in cortical L2/3-Online Resource Fig. 3a). The demyelinated lesions extended approximately $250 \mu \mathrm{m}$ from the cortical surface into L2/3 and were an average of $654 \mu \mathrm{m}$ wide at 2 weeks 
a
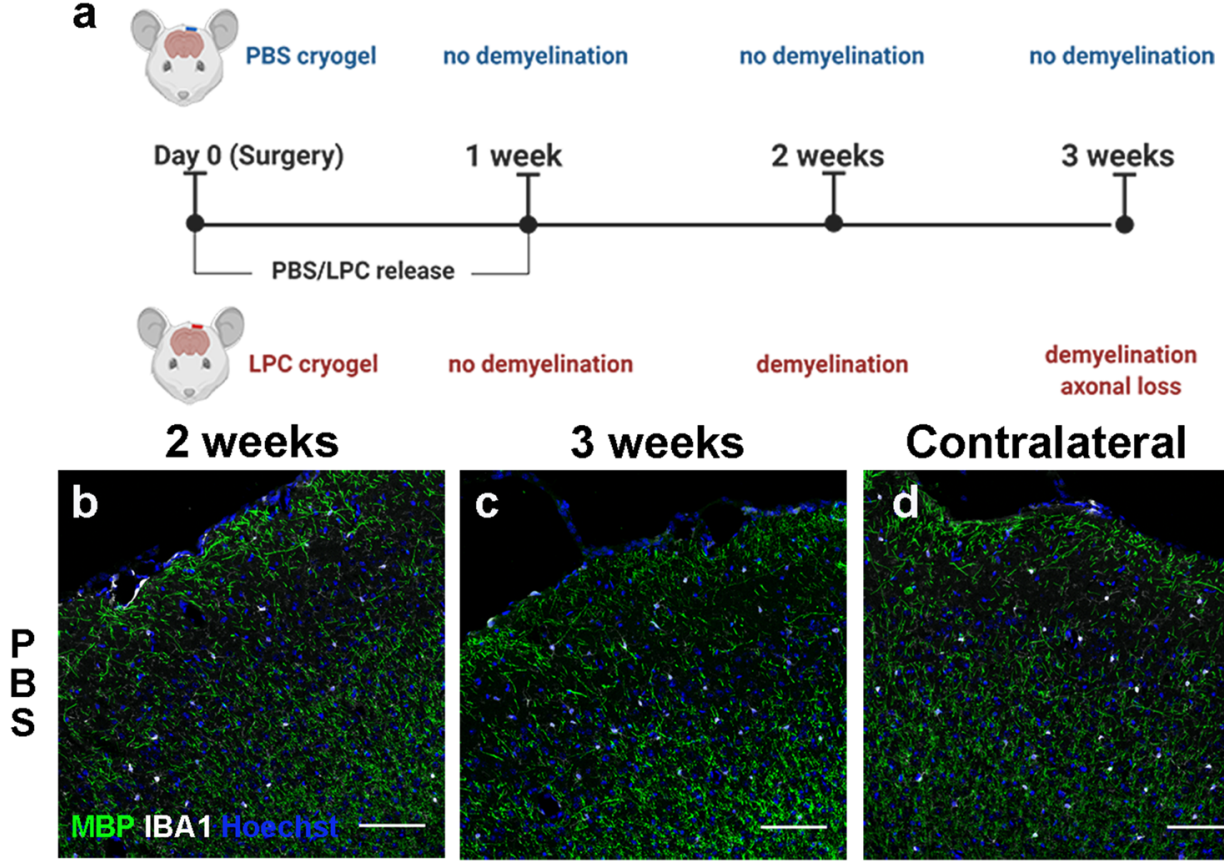

Contralateral
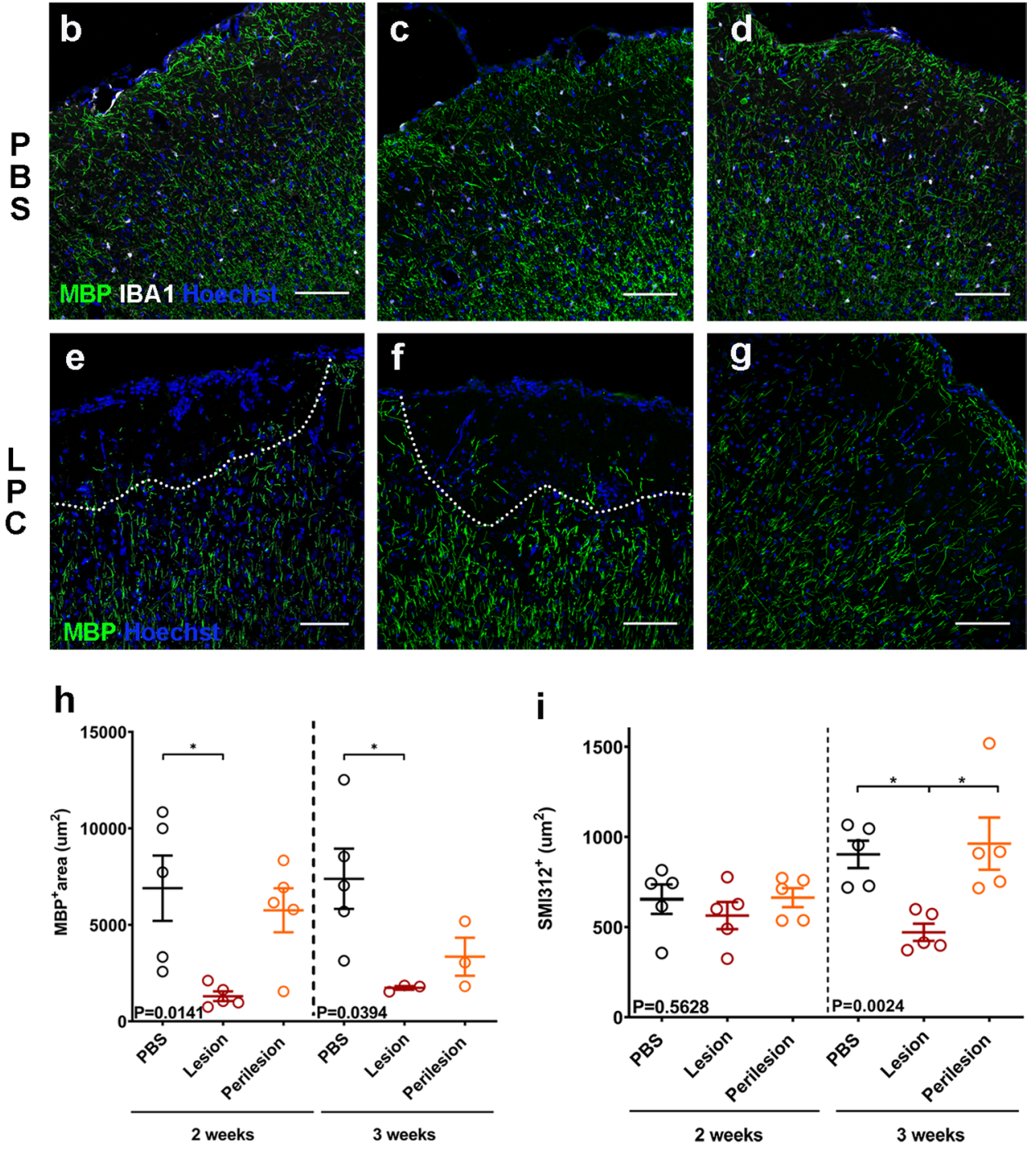

post-surgery (Online Resource Fig. 3c, d). In both lesion and perilesion sites, there were increased numbers of ionized calcium binding adaptor molecule 1 (IBA1)-positive microglia/macrophages (Online Resource Fig. 3e, g, i) and glial fibrillary acidic protein (GFAP)-positive astrogliosis (Online Resource Fig. 3j, 1, n). No effects on gliosis or myelin were observed in the contralateral hemisphere

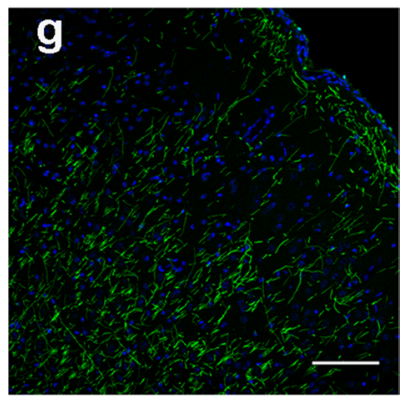

(Fig. 2d, g, Online Resource Fig. 3b, f, h) or when PBSloaded cryogels were applied (Fig. 2b, c \& Online Resource Fig $3 \mathrm{k}, \mathrm{m}$ ). At 3 weeks post-surgery, the focal demyelination persisted (Fig. $2 \mathrm{f}, \mathrm{h}$ ), but with a significant reduction of microglia in the lesion compared to 2 weeks post-surgery (Online Resource Fig. 3g, i) and an attenuation of astrogliosis (Online Resource Fig. 31, n). Axonal loss was not 
४Fig. 2 a Outline of the experimental design. b-d Immunohistochemistry of PBS cryogel treated animals for MBP (green), IBA1 (white) with Hoechst (blue), two (b) and three weeks (c) after cryogel placement. Contralateral hemisphere of the two week, PBS-loaded cryogel (d). Scale bar: $100 \mu \mathrm{m}$. e-g Immunohistochemistry of LPC cryogel treated animals for MBP (green) with Hoechst (blue) two (e) and three weeks (f) after cryogel placement (lesions outlined with dashed lines). Contralateral hemisphere of the two week, LPC-loaded cryogel (g). Scale bar: $100 \mu \mathrm{m}$. h Quantification of MBP + signal at the upper cortical layers of PBS treated cortex (control) LPC-treated lesion and perilesion areas, two (left) and three (right) weeks post -surgery (2 weeks PBS: mean $6900 \pm 1692$ SEM $\mu \mathrm{m}^{2}, N=5$, Lesion: mean $1302 \pm 250.2$ SEM $\mu \mathrm{m}^{2}$, Perilesion: mean $5756 \pm 1139 \mathrm{SEM} \mu \mathrm{m}^{2}, N=5$; 3 weeks PBS: mean $7388 \pm 1561$ SEM $\mu \mathrm{m}^{2}, N=5$, Lesion: mean $1733 \pm 99.51$ SEM $\mu \mathrm{m}^{2}$, Perilesion: mean $3349 \pm 984.8$ SEM $\mu \mathrm{m}^{2}, N=3$; each point is an animal, One-way ANOVA). i Quantification of SMI312+signal at the upper cortical layers of PBS treated cortex (control) LPCtreated lesion and perilesion areas, two (left) and three (right) weeks post -surgery ( 2 weeks PBS: mean $947.1 \pm 63.17 \mathrm{SEM} \mu \mathrm{m}^{2}, N=5$, Lesion: mean $787.8 \pm 52.70$ SEM $\mu \mathrm{m}^{2}$, Perilesion: mean $976.3 \pm 101.3$

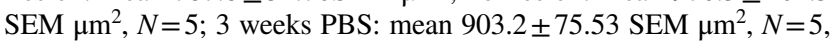
Lesion: mean $471.1 \pm 47.52$ SEM $\mu \mathrm{m}^{2}$, Perilesion: mean $962.9 \pm 144.8$ SEM $\mu \mathrm{m}^{2}, N=5$; each point is an animal, Kruskal-Wallis test)

marked until at 3 weeks post-surgery (approximately 50\% reduction compared to controls) (Fig. 2i). Very few CD3expressing T-cells were seen in this model, restricted only to the area underneath the LPC-loaded cryogel at 2 weeks and subsiding by 3 weeks post-surgery (Online Resource Fig. 3o, p and q).

Using our model, we asked if demyelination can directly lead to selective neurodegeneration. We found a reduced density of PV + interneurons at 2 weeks post-surgery in the LPC treated motor cortex, affecting both lesion and perilesion areas (Fig. 3a, b and c) and this persisted at 3 weeks post-surgery (Fig. 3d). Additionally, we assessed damage to $\mathrm{PV}+$ fibers (axons, dendrites) by quantifying the area covered by PV + puncta, excluding PV + cell bodies, (Fig. 3e, f) which revealed a marked loss of inhibitory fibers in both lesion and perilesion areas by 3 weeks post-surgery (Fig. 3g, h), accounting for the observed total axonal loss seen in Fig. 2i. In contrast, LPC-induced focal subpial demyelination did not alter the densities of vesicular glutamate transporter 2 (VGLUT2) - expressing excitatory pyramidal neurons in cortical L2/3 when compared to PBS controls at 2 or 3 weeks post-surgery (Fig. 3i, j, k and 1); consistent with the human post-mortem data.

The impact of demyelination on the inhibitory network was further corroborated by a differential effect on the density of inhibitory (Fig. 4a, b) and excitatory synapses (Fig. 4. e, f) between PBS control and LPC-lesioned animals at 2 and 3 weeks post-surgery. Fewer inhibitory synapses were detected in the demyelinated lesions by 3 weeks post-surgery compared to PBS controls as measured by automated quantification of the density of overlapping pre-synaptic VGAT and post-synaptic GEPHYRIN puncta (Fig. 4d), with the reduction initiating at 2 weeks (Fig. 4c). The reduction of the inhibitory synapses was attributed to a loss of pre-synaptic components, evident in both lesion and perilesion sites (Online Resource Fig. 4c, d). However, excitatory synapses (overlapping presynaptic vesicular glutamate transporter 1 (VGLUT1) and post-synaptic PSD95 from PSD95-GFP reporter transgenic mouse) did not differ at either time point between LPC-lesion, perilesion and control cortex (Fig. 4g, h\& Online Resource Fig. 4a, b).

In summary, the generation of a single and focal subpial demyelinated cortical lesion in the mouse motor cortex caused a significant and early reduction in the numbers of $\mathrm{PV}+$ interneuron somata and corresponding fibers and synapses, but without any change in excitatory neuron number and synaptic density. Our results successfully replicate the pattern of neurodegeneration seen in the human MS motor cortex and show that focal demyelination can lead to this neurodegeneration.

\section{Functional loss of inhibition is the earliest event after lesion formation}

Histological evidence from our mouse model indicates a selective effect of demyelination on inhibitory neural components. We next asked whether this translated into a functional impairment of neuronal circuitry. To determine the functional consequences of these structural changes, we performed whole-cell patch-clamp recordings from L2 neurons within the LPC-induced demyelinated lesion in the motor cortex compared to PBS controls at 2 weeks post-surgery. To assess the formation of functional synapses, we recorded miniature excitatory and inhibitory postsynaptic currents (mEPSC and mIPSC) recorded at $-70 \mathrm{mV}$ and $0 \mathrm{mV}$, respectively, in the presence of $500 \mathrm{nM}$ tetrodotoxin (TTX, Fig. 4i). We could reliably identify mEPSCs, resulting from putative AMPA receptor-mediated excitatory synapses and mIPSCs, arising from putative GABAA receptor-mediated inhibitory synapses, which reflect synapses located closest to the soma [19]. The properties of mEPSCs was similar to previous reports from L2/3 of the motor cortex[29]. Consistent with our histological analysis, we observed no difference in the amplitude (Fig. 41), frequency (Fig. 4m) or charge-transfer (Fig. 4o) of mEPSCs in L2 neurons from demyelinated or control animals. To assess the function of inhibitory synapses, we next assessed mIPSC properties. In neurons from demyelinated lesions at 2 weeks post-surgery, there was a significant $25 \%$ decrease in mIPSC amplitude (Fig. 41), which was accompanied by a $32 \%$ decrease in mIPSC frequency (Fig. $4 \mathrm{~m}$ ). Together, this resulted in a net $44 \%$ reduction in total inhibitory charge (Fig. 4n), consistent with a major loss of inhibitory synapses. These data verify a functional effect, consistent with of the observed inhibitory synapse loss after demyelination. 


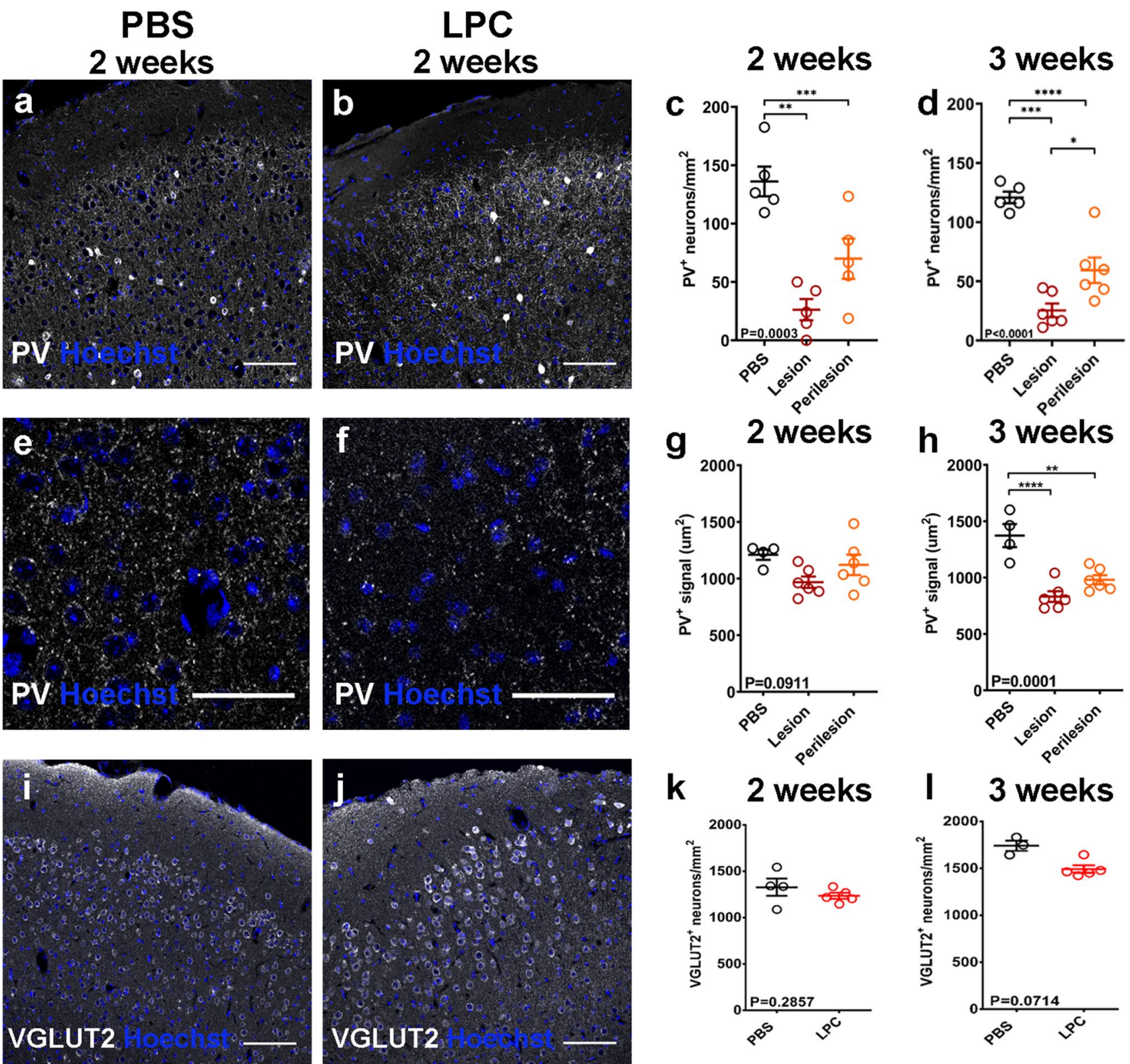

Fig. 3 a and b Immunohistochemistry of PBS (a) and LPC (b) treated animals for PV (white) with Hoechst (blue) two weeks postsurgery. Scale bar: $100 \mu \mathrm{m}$. c-d Quantification of PV+neurons in L2/3 of PBS and LPC-treated animals two weeks (c, PBS: mean $136.1 \pm 12.71$ SEM neurons $/ \mathrm{mm}^{2}, N=5$, Lesion: mean $12.71 \pm 9.107$ SEM neurons $/ \mathrm{mm}^{2}$, Perilesion: mean $69.96 \pm 17.23$ SEM neurons/ $\mathrm{mm}^{2}, N=5$; each point is an animal, One-way ANOVA) and three weeks post-surgery (d, PBS: mean $120.8 \pm 4.876$ SEM neurons $/ \mathrm{mm}^{2}$, $N=5$, Lesion: mean $25.46 \pm 5.756 \mathrm{SEM}$ neurons $/ \mathrm{mm}^{2}$, Perilesion: mean $59.35 \pm 10.80 \mathrm{SEM}$ neurons $/ \mathrm{mm}^{2}, N=6$; each point is an animal, One-way ANOVA). e-f Immunohistochemistry of PBS (e) and LPC (f) treated animals for PV (white) with Hoechst (blue) focusing on $\mathrm{PV}+$ puncta at $\mathrm{L} 2 / 3$, two weeks post-surgery. Scale bar: $50 \mu \mathrm{m}$. g-h Quantification of PV + area in L2/3 of PBS and LPC-treated animals two weeks (g, PBS: mean $1211 \pm 45.12$ SEM $\mu \mathrm{m}^{2}, N=3$,
Lesion: mean $831.7 \pm 47.26$ SEM $\mu \mathrm{m}^{2}$, Perilesion: $981.0 \pm 40.64$ SEM $\mu \mathrm{m}^{2}, N=6$; each point is an animal, One-way ANOVA) and three weeks post-surgery (h, PBS: mean $1373 \pm 102.4$ SEM $\mu \mathrm{m}^{2}$, $N=4$, Lesion: mean $968.1 \pm 50.00 S E M \quad \mu \mathrm{m}^{2}$, Perilesion: mean $1121 \pm 90.13 \mathrm{SEM} \mu \mathrm{m}^{2}, N=6$, each point is an animal, One-way ANOVA). i-j Immunohistochemistry of PBS (i) and LPC (j) treated animals for VGLUT2 (white) with Hoechst (blue) two weeks postsurgery. Scale bar: $100 \mu \mathrm{m}$. k-l Quantification of VGLUT2 + neurons in L2/3 of PBS and LPC-treated animals two weeks (k, PBS: mean $1328 \pm 93.33$ SEM neurons $/ \mathrm{mm}^{2}, N=4$, LPC: mean $1234 \pm 31.72$ SEM neurons $/ \mathrm{mm}^{2}, N=5$; each point is an animal, Mann Whitney test) and three weeks post-surgery (1, PBS: mean $1741 \pm 54.65$ SEM neurons $/ \mathrm{mm}^{2}, N=3$, LPC: mean $1492 \pm 39.51$ SEM neurons $/ \mathrm{mm}^{2}$, $N=5$; each point is an animal, Mann Whitney test) 
These electrophysiological recordings confirm that focal subpial grey matter demyelination is sufficient to cause functional loss of inhibitory synapses with no change in excitation in the cortex, in line with the selective anatomical neurodegeneration of the inhibitory components.

\section{Discussion}

We have identified a distinct neurodegenerative signature in the secondary progressive MS motor cortex characterized by the selective loss of interneuron subtypes and their connections. In a preclinical mouse model, we showed that this selective vulnerability is secondary to cortical demyelination and affects $\mathrm{PV}+$, fast-spiking interneurons. The anatomical loss of $\mathrm{PV}+$ interneurons is quickly translated into a functional neurophysiological impairment of the inhibitory circuitry, providing a link between grey matter demyelination and selective neurodegeneration of inhibitory neuronal components.

The selective effect on the inhibitory network in the human progressive MS motor cortex opposes the initial hypothesis that MS-induced neurodegeneration affects all neuronal types equally. Although at post-mortem we can only study the end-stage of a chronic and progressive disease, our results indicate that the inhibitory neural network is more susceptible to damage and/or less receptive to repair than the excitatory one. This is in direct contrast to a recent article showing no loss of PV neurons but instead a loss of the upper layer CUX2-expressing projection neurons in MS tissue, using snRNAseq [40]. This may simply be as these samples were combinations of white matter and grey matter with PV + cells forming less than $2 \%$ of the total cells so that population changes in this low number of cells may have been missed. We saw no significant change in CUX2 + cells using a CUX2 antibody in our samples, rather than a $C U X 2$ RNA probe (as used in [40]). It is worth noting that the $C U X 2$ transcript is expressed in the $\mathrm{PV}$ interneuron cluster as well as in the excitatory neurons, therefore, the loss seen may be related to the loss of some $\mathrm{PV}+$ neurons.

Post-mortem analysis cannot explain the exact role of demyelination in MS neurodegeneration as there is no temporal data and inflammatory damage or excitotoxic damage due to neuronal network dysfunction still remain potential contributors to the observed interneuron vulnerability [15, 31]. As our findings are very similar between demyelinated and normal appearing grey matter, it is possible that the latter is not normal, either due to damage followed by remyelination (which is difficult to distinguish due to sparse cortical myelination) or as perilesional areas (with the demyelinated lesion excluded from the sampled section due to tissue size). Therefore, to understand if demyelination was the cause of the selective neurodegeneration in human motor cortex, the development of our mouse model was necessary.

Our preclinical model complements existing other rodent models of cortical demyelination: focal EAE (experimental allergic encephalomyelitis) and global cuprizone. Our model permits the generation of a focal subpial cortical lesion of controlled and limited size, in contrast to the global cortical and white matter demyelination observed in the cuprizone model [34] and avoids T-cell driven inflammatory demyelination, provoked in EAE [35]). It successfully models the subpial lesions of cortical demyelination also seen in human post-mortem MS tissue and it is well tolerated.

A single focal demyelinated lesion in the superficial cortical layers of mouse replicated the loss of interneuron somata, axons and their inhibitory synapses, as observed in the human tissue and resulted in a functional impairment of inhibitory transmission. PV + interneurons, PV + fibers and inhibitory synapses were very quickly lost after demyelination which led us to the question as to why inhibitory interneurons are more at risk than pyramidal neurons after cortical demyelination. Part of the answer may rely on the location of myelinated neurons, the amount of myelination per axon and the importance of myelination for their metabolic support.

Excitatory pyramidal neurons have their soma in a specific cortical layer and send their axons over long distances, often innervating distant brain areas. Many also show discontinuous patterns of myelination [45]. Conversely, most cortical interneurons have short but elaborate axonal processes that reside in the same cortical area [17]. A large fraction of cortical interneuron axons is myelinated in rodents and humans, the majority of which belong to the PV + basket cells, the most abundant cortical interneuron subtype [37, 38, 42, 43, 51]. In mouse, approximately $65 \%$ of each PV + axon is covered by myelin compared to approximately $25 \%$ for SST + and $10 \%$ for vasoactive intestinal polypeptide expressing interneurons suggesting that these different myelination patterns reflect nuances in the importance of myelination for each neuronal subtype [51]. Heavily myelinated PV + basket cells directly target the soma and proximal dendrites of pyramidal neurons and other interneurons mediating fast rhythmic inhibition, which requires a constant supply of ATP as manifested by their increased numbers of axonal and pre-synaptic mitochondria and cytochrome $c$ oxidase $[11,20]$. This implies that PV + axons are more reliant on the metabolic coupling between the axon and the oligodendrocyte for nutrient supply. Indeed, previous studies in which the cytochrome c oxidase pathway was ablated showed preferential deficits in the recruitment of inhibition suggesting a causal link [5]. Given the location of these cells, their locally projecting axons and their dependence on myelination it is reasonable to speculate that the relative amount of myelin 

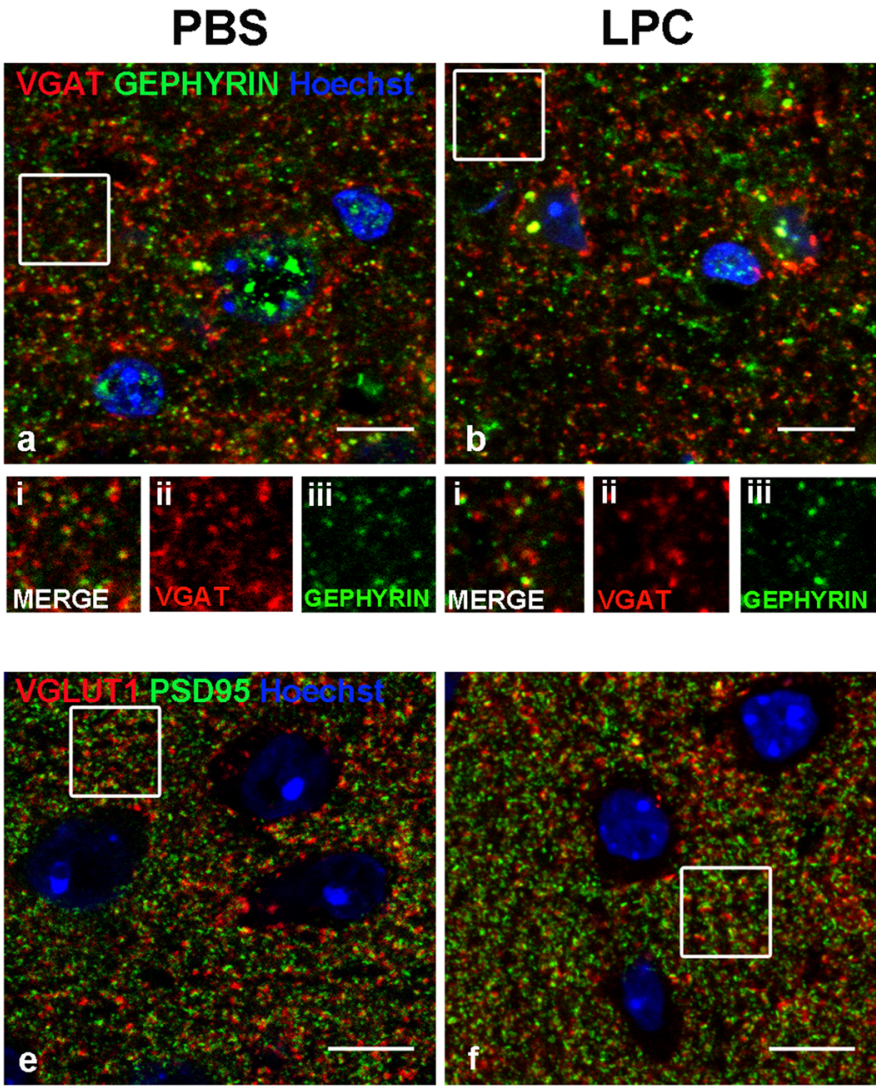
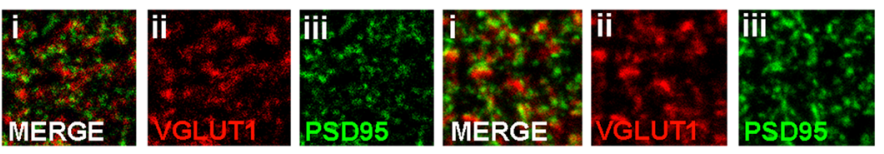

i

mIPSC

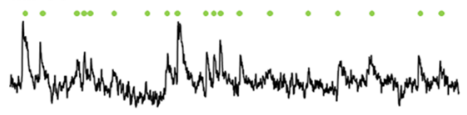
mEPSC

(1) mIPSC

LPC cryogel

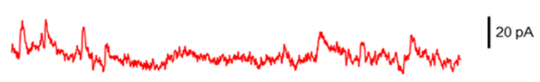
mEPSC

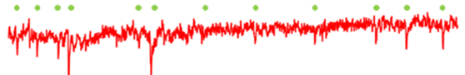

$$
\varlimsup_{100 \mathrm{~ms}}^{10 \mathrm{pA}}
$$

j
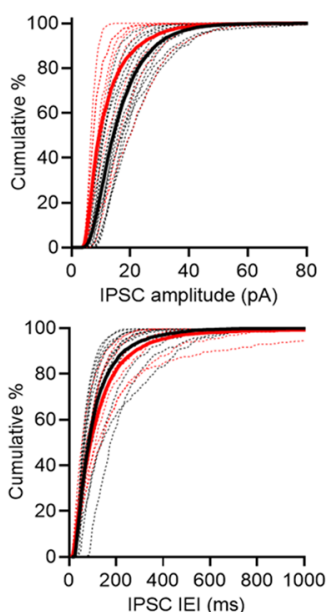

Inhibitory Synapses
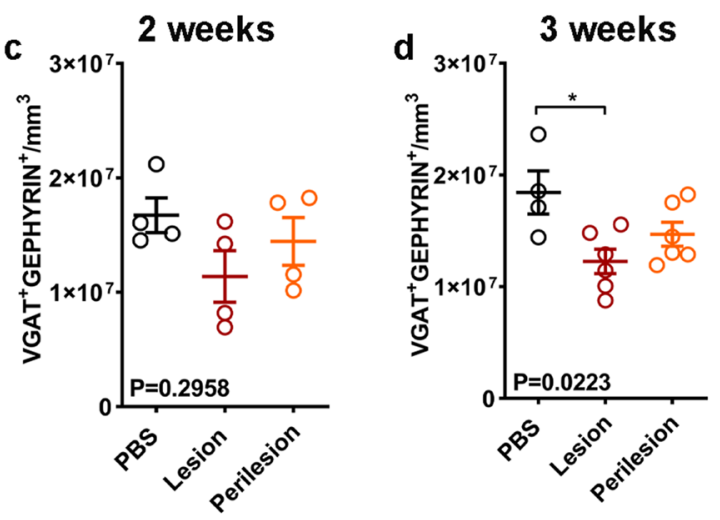

Excitatory Synapses
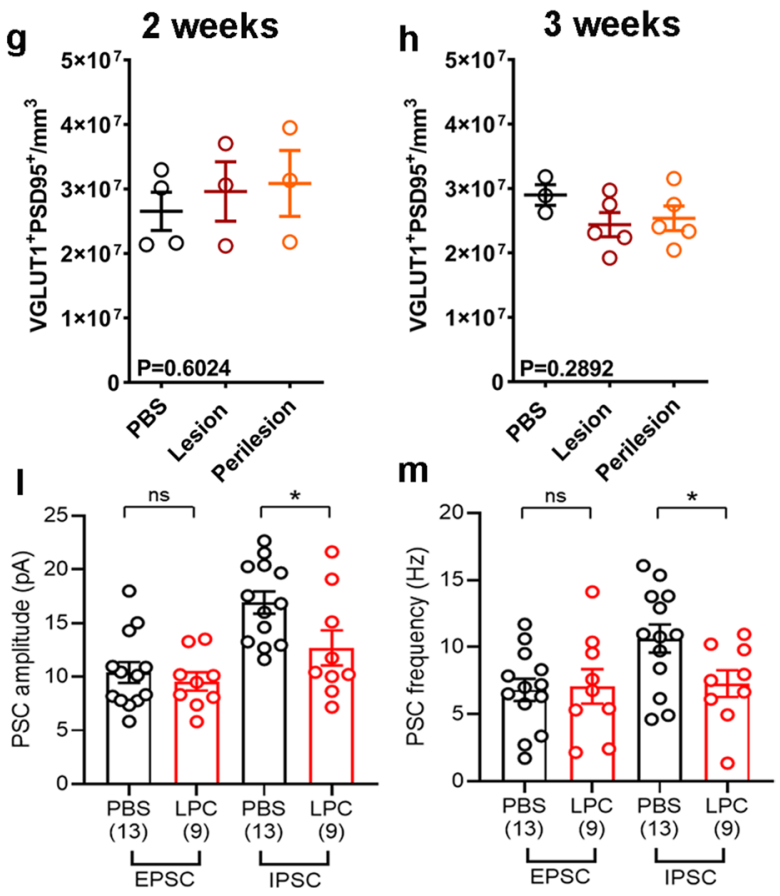

n

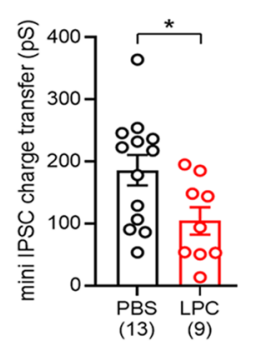

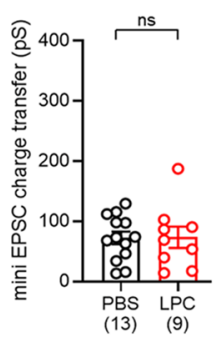

lost per axon is higher for PV + interneuron axons than pyramidal ones, which renders them more susceptible to irreversible damage when the superficial cortical layers are demyelinated.

In the mouse model, the fast loss of PV + interneurons after demyelination coincided with a marked accumulation of microglia and increased astrogliosis at the lesion site. Although no reactivity of either cell type was observed prior to demyelination in our model, in contrast to what was observed previously for EAE [48] and that LPC-induced demyelination is known to provoke a microglial and astroglial response, these responses may also contribute to the overall interneuron damage after demyelination. The fast loss of $\mathrm{PV}+$ interneurons also resulted in a marked reduction 
४Fig. 4 a and b Immunohistochemistry of PBS (a) and LPC (b) treated animals for VGAT (red), GEPHYRIN (green) with Hoechst (blue) two weeks post-surgery. i-iii: Inset images of boxed areas in $a$ or b showing merged (i) and single channels of the synaptic proteins VGAT (ii) and GEPHYRIN (iii). Scale bar: $10 \mu \mathrm{m}$. c-d Quantification of VGAT1+/GEPHYRIN + inhibitory synapses in L2/3 of PBS and LPC-treated animals two (c, PBS: mean $1.673 \mathrm{e}+007 \pm 1.520 \mathrm{e}+006$ SEM synapses $/ \mathrm{mm}^{3}, \mathrm{~N}=4$, Lesion: mean $1.138 \mathrm{e}+007 \pm 2.255 \mathrm{e}+006$ SEM synapses $/ \mathrm{mm}^{3}$, Perilesion: mean $1.444 \mathrm{e}+007 \pm 2.092 \mathrm{e}+006 \mathrm{SEM}$ synapses $/ \mathrm{mm}^{3}, \mathrm{~N}=4$; each point is an animal, Kruskal-Wallis test) and three weeks postsurgery (d, PBS: mean $1.844 \mathrm{e}+007 \pm 1.936 \mathrm{e}+006$ SEM synapses/ $\mathrm{mm}^{3}, \mathrm{~N}=4$, Lesion: mean $1.226 \mathrm{e}+007 \pm 1.088 \mathrm{e}+006$ SEM synapses $/ \mathrm{mm}^{3}$, Perilesion: mean $1.469 \mathrm{e}+007 \pm 1.072 \mathrm{e}+006$ SEM synapses $/ \mathrm{mm}^{3}, \mathrm{~N}=6$; each point is an animal, One-way ANOVA). e-f Immunohistochemistry of PBS (e) and LPC (f) treated animals for VGLUT1 (red), PSD95 (green) with Hoechst (blue) two weeks postsurgery. i-iii: Inset images of boxed areas in e or f showing merged (i) and single channels of the synaptic proteins VGLUT1 (ii) and PSD95 (iii). Scale bar: $10 \mu \mathrm{m}$. g-h Quantification of VGLUT1+/ PSD95 + excitatory synapses in L2/3 of PBS and LPC-treated animals two weeks (g, PBS: mean $2.653 \mathrm{e}+007 \pm 2.963 \mathrm{e}+006$ SEM synapses $/ \mathrm{mm}^{3}, \quad \mathrm{~N}=4$, Lesion: mean $2.961 \mathrm{e}+007 \pm 4.605 \mathrm{e}+006$ SEM synapses $/ \mathrm{mm}^{3}$, Perilesion: mean 3.086e $+007 \pm 5.115 \mathrm{e}+006$ SEM synapses $/ \mathrm{mm}^{3}, \mathrm{~N}=3$; each point is an animal, KruskalWallis test) and three weeks post-surgery (h, PBS: mean $2.899 \mathrm{e}+007 \pm 1.597 \mathrm{e}+006$ SEM synapses $/ \mathrm{mm}^{3}, \mathrm{~N}=3$, Lesion: mean $2.438 \mathrm{e}+007 \pm 1.875 \mathrm{e}+006$ SEM synapses $/ \mathrm{mm}^{3}$, Perilesion: mean $2.4536 \mathrm{e}+007 \pm 1.908 \mathrm{e}+006 \mathrm{SEM}$ synapses $/ \mathrm{mm}^{3}, \mathrm{~N}=5$; each point is an animal, Kruskal-Wallis test). i: Representative miniature inhibitory postsynaptic currents (mIPSC) and miniature excitatory postsynaptic currents (mEPSC) recorded at $0 \mathrm{mV}$ and $-70 \mathrm{mV}$ respectively in the presence of $500 \mathrm{nM}$ TTX, from L2 pyramidal cells from the motor cortex, 2 weeks post-surgery. Individual detected miniature events are indicated (green dots). Data are shown from representative cells from PBS (upper, black) and LPC (lower, red) treated mice. j: Cumulative distribution of mIPSC amplitudes from PBS (black) and LPC (red) treated mice, recorded at 2 weeks following surgery. Individual distributions from each neuron (thin dashed lines) are shown underlying the mean distribution (thick lines). k: Cumulative distributions of IPSC inter-event interval (IEI) recorded at 2 weeks postsurgery, plotted according to the same scheme as j. l: Amplitudes of mEPSC and mIPSCs recorded from L2 pyramidal neurons in PBS (black) and LPC (red) treated mice at 2 weeks post-surgery (5 PBS mice and 3 LPC mice). Number of recorded neurons is indicated. m: Frequencies of mEPSC and mIPSCs recorded from 12 pyramidal neurons in PBS (black) and LPC (red) treated mice at 2 weeks postsurgery (5 PBS mice and 3 LPC mice). Number of recorded neurons is indicated. n: Total charge transferred by mIPSCs recorded at $0 \mathrm{mV}$ for $5 \mathrm{~min}$ at 2 weeks post-surgery (5 PBS mice and 3 LPC mice). Number of recorded neurons is indicated. o: Total charge transferred by mEPSCs recorded at $-70 \mathrm{mV}$ over $5 \mathrm{~min}$ of recording from PBS and LPC treated mice at 2 weeks post-surgery (5 PBS mice and 3 LPC mice). Number of recorded neurons is indicated. Statistics shown non-significance (ns) $-p>.05, *-p<.05$, unpaired t-tests with Welch's correction

in the frequency, amplitude and charge transfer of inhibitory post-synaptic currents albeit with no effect on excitatory post synaptic currents. This functional impairment of inhibitory transmission observed in rodents is of relevance to human MS. In the healthy human motor cortex, GABAergic neurotransmission is related to motor learning and use-dependent plasticity $[28,41]$. In secondary progressive MS, GABA levels were found reduced in the somatosensory cortex of patients and were linked to reduced motor performance [9, 47]. We now provide evidence that this selective neurodegeneration observed in both our rodent model and human MS can be secondary to cortical demyelination, primarily through loss of PV + interneurons, their axons and synapses. This new understanding of the pattern of cortical neurodegeneration in MS allows us to think more strategically about neuroprotective therapies, perhaps by providing increased and targeted support to PV + interneurons and selectively increasing their remyelination. Our new preclinical mouse model gives us a tractable relevant system to carry out drug screens to test this strategy with the aim of developing new treatments for progressive MS.

Acknowledgements The authors would like to thank and acknowledge the Edinburgh Brain and Tissue Bank and the MS Society UK Tissue Bank for providing tissues used in this study. The Edinburgh Brain Bank is supported by the Medical Research Council, ethical approval NHS-RECSE (16/ES/0084). We thank Prof. Seth Grant for giving us the PSD95-eGFP transgenic mouse. LZ is funded by the MS Society UK Centre grant to Edinburgh. AW is also funded by the MS Society UK and the Medical Research Council. TSJ is funded by UK Dementia Research Institute which receives funding from Alzheimer's Research UK, the Alzheimer's Society and the Medical Research Council and the European Research Council (ERC) under the European Union's Horizon 2020 research and innovation programme (Grant agreement No. 681181). BN, CW and DE are funded by the Deutsche Forschungsgemeinschaft (DFG) - Project number 320041273. SAB and PK are funded by the Simons Foundation Autism Research Initiative (529085) and the Patrick Wild Centre. We would like to thank Rosie Jackson and Chris Henstridge for their help in array tomography processing and analysis and Dr. Veronique Miron, Prof. David Lyons and Prof. Charles ffrench-Constant for their comments on the manuscript.

Open Access This article is licensed under a Creative Commons Attribution 4.0 International License, which permits use, sharing, adaptation, distribution and reproduction in any medium or format, as long as you give appropriate credit to the original author(s) and the source, provide a link to the Creative Commons licence, and indicate if changes were made. The images or other third party material in this article are included in the article's Creative Commons licence, unless indicated otherwise in a credit line to the material. If material is not included in the article's Creative Commons licence and your intended use is not permitted by statutory regulation or exceeds the permitted use, you will need to obtain permission directly from the copyright holder. To view a copy of this licence, visit http://creativecommons.org/licenses/by/4.0/.

\section{References}

1. Albert M, Barrantes-Freer A, Lohrberg M, Antel JP, Prineas JW, Palkovits M, Wolff JR, Brück W, Stadelmann C (2017) Synaptic pathology in the cerebellar dentate nucleus in chronic multiple sclerosis. Brain Pathol 27:737-747. https://doi.org/10.1111/ bpa. 12450

2. Bankhead P, Loughrey MB, Fernández JA, Dombrowski Y, McArt DG, Dunne PD, McQuaid S, Gray RT, Murray LJ, Coleman HG, James JA, Salto-Tellez M, Hamilton PW (2017) QuPath: Open 
source software for digital pathology image analysis. Sci Rep 7:16878. https://doi.org/10.1038/s41598-017-17204-5

3. Bø L, Vedeler CA, Nyland H, Trapp BD, Mørk SJ (2003) Intracortical multiple sclerosis lesions are not associated with increased lymphocyte infiltration. Mult Scler 9:323-331. https://doi. org/10.1191/1352458503ms917oa

4. Bø L, Vedeler CA, Nyland HI, Trapp BD, Mørk SJ (2003) Subpial demyelination in the cerebral cortex of multiple sclerosis patients. J Neuropathol Exp Neurol 62:723-732. https://doi.org/10.1093/ jnen/62.7.723

5. Booker SA, Campbell GR, Mysiak KS, Brophy PJ, Kind PC, Mahad DJ, Wyllie DJ (2017) Loss of protohaem IX farnesyltransferase in mature dentate granule cells impairs short-term facilitation at mossy fibre to CA3 pyramidal cell synapses. J Physiol 595:2147-2160. https://doi.org/10.1113/JP273581

6. Booker SA, Simões de Oliveira L, Anstey NJ, Kozic Z, Dando OR, Jackson AD, Baxter PS, Isom LL, Sherman DL, Hardingham GE, Brophy PJ, Wyllie DJA, Kind PC (2020) Input-Output Relationship of CA1 Pyramidal Neurons Reveals Intact Homeostatic Mechanisms in a Mouse Model of Fragile X Syndrome. Cell Rep 32:107988. https://doi.org/10.1016/j.celrep.2020.107988

7. Calabrese M, Poretto V, Favaretto A, Alessio S, Bernardi V, Romualdi C, Rinaldi F, Perini P, Gallo P (2012) Cortical lesion load associates with progression of disability in multiple sclerosis. Brain 135:2952-2961. https://doi.org/10.1093/brain/aws246

8. Carassiti D, Altmann DR, Petrova N, Pakkenberg B, Scaravilli F, Schmierer K (2018) Neuronal loss, demyelination and volume change in the multiple sclerosis neocortex. Neuropathol Appl Neurobiol 44:377-390. https://doi.org/10.1111/nan.12405

9. Cawley N, Solanky BS, Muhlert N, Tur C, Edden RA, WheelerKingshott CA, Miller DH, Thompson AJ, Ciccarelli O (2015) Reduced gamma-aminobutyric acid concentration is associated with physical disability in progressive multiple sclerosis. Brain 138:2584-2595. https://doi.org/10.1093/brain/awv209

10. Clements JD, Bekkers JM (1997) Detection of spontaneous synaptic events with an optimally scaled template. Biophys J 73:220 229. https://doi.org/10.1016/S0006-3495(97)78062-7

11. Cserép C, Pósfai B, Schwarcz AD, Dénes Á (2018) Mitochondrial Ultrastructure Is Coupled to Synaptic Performance at Axonal Release Sites. eNeuro. https://doi.org/10.1523/ENEUR O.0390-17.2018

12. Dutta R, Chang A, Doud MK, Kidd GJ, Ribaudo MV, Young EA, Fox RJ, Staugaitis SM, Trapp BD (2011) Demyelination causes synaptic alterations in hippocampi from multiple sclerosis patients. Ann Neurol 69:445-454. https://doi.org/10.1002/ ana. 22337

13. Dutta R, McDonough J, Yin X, Peterson J, Chang A, Torres T, Gudz T, Macklin WB, Lewis DA, Fox RJ, Rudick R, Mirnics K, Trapp BD (2006) Mitochondrial dysfunction as a cause of axonal degeneration in multiple sclerosis patients. Ann Neurol 59:478489. https://doi.org/10.1002/ana.20736

14. Eigel D, Zoupi L, Sekizar S, Welzel PB, Werner C, Williams A, Newland B (2019) Cryogel scaffolds for regionally constrained delivery of lysophosphatidylcholine to central nervous system slice cultures: A model of focal demyelination for multiple sclerosis research. Acta Biomater 97:216-229. https://doi.org/10.1016/j. actbio.2019.08.030

15. Ellwardt E, Pramanik G, Luchtman D, Novkovic T, Jubal ER, Vogt J, Arnoux I, Vogelaar CF, Mandal S, Schmalz M, Barger Z, Ruiz de Azua I, Kuhlmann T, Lutz B, Mittmann T, Bittner S, Zipp F, Stroh A (2018) Maladaptive cortical hyperactivity upon recovery from experimental autoimmune encephalomyelitis. Nat Neurosci 21:1392-1403. https://doi.org/10.1038/s41593-018-0193-2

16. Faissner S, Plemel JR, Gold R, Yong VW (2019) Progressive multiple sclerosis: from pathophysiology to therapeutic strategies.
Nat Rev Drug Discov 18:905-922. https://doi.org/10.1038/s4157 3-019-0035-2

17. Fishell G, Kepecs A (2020) Interneuron Types as Attractors and Controllers. Annu Rev Neurosci 43:1-30. https://doi.org/10.1146/ annurev-neuro-070918-050421

18. Gardner C, Magliozzi R, Durrenberger PF, Howell OW, Rundle J, Reynolds R (2013) Cortical grey matter demyelination can be induced by elevated pro-inflammatory cytokines in the subarachnoid space of MOG-immunized rats. Brain 136:3596-3608. https ://doi.org/10.1093/brain/awt279

19. Glickfeld LL, Atallah BV, Scanziani M (2008) Complementary modulation of somatic inhibition by opioids and cannabinoids. J Neurosci 28:1824-1832. https://doi.org/10.1523/JNEUR OSCI.4700-07.2008

20. Gulyás AI, Buzsáki G, Freund TF, Hirase H (2006) Populations of hippocampal inhibitory neurons express different levels of cytochrome c. Eur J Neurosci 23:2581-2594. https://doi.org/10 $.1111 / \mathrm{j} .1460-9568.2006 .04814 . x$

21. Guzman SJ, Schlögl A, Schmidt-Hieber C (2014) Stimfit: quantifying electrophysiological data with Python. Front Neuroinform 8:16. https://doi.org/10.3389/fninf.2014.00016

22. Harrison DM, Roy S, Oh J, Izbudak I, Pham D, Courtney S, Caffo B, Jones CK, van Zijl P, Calabresi PA (2015) Association of Cortical Lesion Burden on 7-T Magnetic Resonance Imaging With Cognition and Disability in Multiple Sclerosis. JAMA Neurol 72:1004-1012. https://doi.org/10.1001/jamaneurol.2015.1241

23. Junker A, Wozniak J, Voigt D, Scheidt U, Antel J, Wegner C, Brück W, Stadelmann C (2020) Extensive subpial cortical demyelination is specific to multiple sclerosis. Brain Pathol. https://doi. org/10.1111/bpa.12813

24. Jürgens T, Jafari M, Kreutzfeldt M, Bahn E, Brück W, Kerschensteiner M, Merkler D (2016) Reconstruction of single cortical projection neurons reveals primary spine loss in multiple sclerosis. Brain 139:39-46. https://doi.org/10.1093/brain/awv353

25. Kay KR, Smith C, Wright AK, Serrano-Pozo A, Pooler AM, Koffie R, Bastin ME, Bak TH, Abrahams S, Kopeikina KJ, McGuone D, Frosch MP, Gillingwater TH, Hyman BT, SpiresJones TL (2013) Studying synapses in human brain with array tomography and electron microscopy. Nat Protoc 8:1366-1380. https://doi.org/10.1038/nprot.2013.078

26. Kidd D, Barkhof F, McConnell R, Algra PR, Allen IV, Revesz T (1999) Cortical lesions in multiple sclerosis. Brain 122(Pt 1):1726. https://doi.org/10.1093/brain/122.1.17

27. Kornek B, Storch MK, Weissert R, Wallstroem E, Stefferl A, Olsson T, Linington C, Schmidbauer M, Lassmann H (2000) Multiple sclerosis and chronic autoimmune encephalomyelitis: a comparative quantitative study of axonal injury in active, inactive and remyelinated lesions. Am J Pathol 157:267-276. https://doi. org/10.1016/S0002-9440(10)64537-3

28. Levy LM, Ziemann U, Chen R, Cohen LG (2002) Rapid modulation of GABA in sensorimotor cortex induced by acute deafferentation. Ann Neurol 52:755-761. https://doi.org/10.1002/ ana.10372

29. Li H, Prince DA (2002) Synaptic activity in chronically injured, epileptogenic sensory-motor neocortex. J Neurophysiol 88:2-12. https://doi.org/10.1152/jn.00507.2001

30. Lovato L, Willis SN, Rodig SJ, Caron T, Almendinger SE, Howell OW, Reynolds R, O'Connor KC, Hafler DA (2011) Related B cell clones populate the meninges and parenchyma of patients with multiple sclerosis. Brain 134:534-541. https://doi.org/10.1093/ brain/awq350

31. Lucchinetti CF, Popescu BF, Bunyan RF, Moll NM, Roemer SF, Lassmann H, Brück W, Parisi JE, Scheithauer BW, Giannini C, Weigand SD, Mandrekar J, Ransohoff RM (2011) Inflammatory cortical demyelination in early multiple sclerosis. N Engl J Med 365:2188-2197. https://doi.org/10.1056/NEJMoa1100648 
32. Magliozzi R, Howell OW, Durrenberger P, Aricò E, James R, Cruciani C, Reeves C, Roncaroli F, Nicholas R, Reynolds R (2019) Meningeal inflammation changes the balance of TNF signalling in cortical grey matter in multiple sclerosis. J Neuroinflammation 16:259. https://doi.org/10.1186/s12974-019-1650-x

33. Magliozzi R, Scalfari A, Pisani AI, Ziccardi S, Marastoni D, Pizzini FB, Bajrami A, Tamanti A, Guandalini M, Bonomi S, Rossi S, Mazziotti V, Castellaro M, Montemezzi S, Rasia S, Capra R, Pitteri M, Romualdi C, Reynolds R, Calabrese M (2020) The CSF Profile Linked to Cortical Damage Predicts Multiple Sclerosis Activity. Ann Neurol. https://doi.org/10.1002/ana.25786

34. Matsushima GK, Morell P (2001) The neurotoxicant, cuprizone, as a model to study demyelination and remyelination in the central nervous system. Brain Pathol 11:107-116. https://doi. org/10.1111/j.1750-3639.2001.tb00385.x

35. Merkler D, Ernsting T, Kerschensteiner M, Brück W, Stadelmann C (2006) A new focal EAE model of cortical demyelination: multiple sclerosis-like lesions with rapid resolution of inflammation and extensive remyelination. Brain 129:1972-1983. https://doi. org/10.1093/brain/awl135

36. Michailidou I, Willems JG, Kooi EJ, van Eden C, Gold SM, Geurts JJ, Baas F, Huitinga I, Ramaglia V (2015) Complement C1q-C3-associated synaptic changes in multiple sclerosis hippocampus. Ann Neurol 77:1007-1026. https://doi.org/10.1002/ ana. 24398

37. Micheva KD, Chang EF, Nana AL, Seeley WW, Ting JT, Cobbs C, Lein E, Smith SJ, Weinberg RJ, Madison DV (2018) Distinctive Structural and Molecular Features of Myelinated Inhibitory Axons in Human Neocortex. eNeuro. https://doi.org/10.1523/ ENEURO.0297-18.2018

38. Micheva KD, Wolman D, Mensh BD, Pax E, Buchanan J, Smith SJ, Bock DD (2016) A large fraction of neocortical myelin ensheathes axons of local inhibitory neurons. Elife. https://doi. org/10.7554/eLife. 15784

39. Peterson JW, Bö L, Mörk S, Chang A, Trapp BD (2001) Transected neurites, apoptotic neurons and reduced inflammation in cortical multiple sclerosis lesions. Ann Neurol 50:389-400. https ://doi.org/10.1002/ana.1123

40. Schirmer L, Velmeshev D, Holmqvist S, Kaufmann M, Werneburg S, Jung D, Vistnes S, Stockley JH, Young A, Steindel M, Tung B, Goyal N, Bhaduri A, Mayer S, Engler JB, Bayraktar OA, Franklin RJM, Haeussler M, Reynolds R, Schafer DP, Friese MA, Shiow LR, Kriegstein AR, Rowitch DH (2019) Neuronal vulnerability and multilineage diversity in multiple sclerosis. Nature 573:7582. https://doi.org/10.1038/s41586-019-1404-z

41. Stagg CJ, Bachtiar V, Johansen-Berg H (2011) The role of GABA in human motor learning. Curr Biol 21:480-484. https://doi. org/10.1016/j.cub.2011.01.069
42. Stedehouder J, Brizee D, Slotman JA, Pascual-Garcia M, Leyrer ML, Bouwen BL, Dirven CM, Gao Z, Berson DM, Houtsmuller AB, Kushner SA (2019) Local axonal morphology guides the topography of interneuron myelination in mouse and human neocortex. Elife. https://doi.org/10.7554/eLife.48615

43. Stedehouder J, Couey JJ, Brizee D, Hosseini B, Slotman JA, Dirven CMF, Shpak G, Houtsmuller AB, Kushner SA (2017) Fastspiking Parvalbumin Interneurons are Frequently Myelinated in the Cerebral Cortex of Mice and Humans. Cereb Cortex 27:50015013. https://doi.org/10.1093/cercor/bhx203

44. Stedehouder J, Kushner SA (2017) Myelination of parvalbumin interneurons: a parsimonious locus of pathophysiological convergence in schizophrenia. Mol Psychiatry 22:4-12. https://doi. org/10.1038/mp.2016.147

45. Tomassy GS, Berger DR, Chen HH, Kasthuri N, Hayworth KJ, Vercelli A, Seung HS, Lichtman JW, Arlotta P (2014) Distinct profiles of myelin distribution along single axons of pyramidal neurons in the neocortex. Science 344:319-324. https://doi. org/10.1126/science. 1249766

46. Trapp BD, Ransohoff R, Rudick R (1999) Axonal pathology in multiple sclerosis: relationship to neurologic disability. Curr Opin Neurol 12:295-302. https://doi.org/10.1097/00019052-19990 6000-00008

47. Vucic S, Burke T, Lenton K, Ramanathan S, Gomes L, Yannikas C, Kiernan MC (2012) Cortical dysfunction underlies disability in multiple sclerosis. Mult Scler 18:425-432. https://doi. org/10.1177/1352458511424308

48. Werneburg S, Jung J, Kunjamma RB, Ha SK, Luciano NJ, Willis CM, Gao G, Biscola NP, Havton LA, Crocker SJ, Popko B, Reich DS, Schafer DP (2020) Targeted Complement Inhibition at Synapses Prevents Microglial Synaptic Engulfment and Synapse Loss in Demyelinating Disease. Immunity 52:167-182.e167. https ://doi.org/10.1016/j.immuni.2019.12.004

49. Witte ME, Mahad DJ, Lassmann H, van Horssen J (2014) Mitochondrial dysfunction contributes to neurodegeneration in multiple sclerosis. Trends Mol Med 20:179-187. https://doi. org/10.1016/j.molmed.2013.11.007

50. Zhu F, Cizeron M, Qiu Z, Benavides-Piccione R, Kopanitsa MV, Skene NG, Koniaris B, DeFelipe J, Fransén E, Komiyama NH, Grant SGN (2018) Architecture of the Mouse Brain Synaptome. Neuron 99:781-799.e710. https://doi.org/10.1016/j.neuro n.2018.07.007

51. Zonouzi M, Berger D, Jokhi V, Kedaigle A, Lichtman J, Arlotta P (2019) Individual Oligodendrocytes Show Bias for Inhibitory Axons in the Neocortex. Cell Rep 27:2799-2808.e2793. https:// doi.org/10.1016/j.celrep.2019.05.018

Publisher's Note Springer Nature remains neutral with regard to jurisdictional claims in published maps and institutional affiliations. 